\title{
Distribution, location, and transcriptional profile of Peyer's patch conventional DC subsets at steady state and under TLR7 ligand stimulation
}

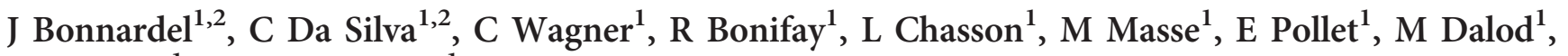 \\ $\mathrm{J}_{-\mathrm{P}}$ Gorvel $^{1}$ and $\mathrm{H}$ Lelouard ${ }^{1}$
}

The initiation of the mucosal immune response in Peyer's patch (PP) relies on the sampling, processing, and efficient presentation of foreign antigens by dendritic cells (DCs). Among PP DCs, CD11b ${ }^{+}$conventional DCs (cDCs) and lysozyme-expressing DCs (LysoDCs) have distinct progenitors and functions but share many cell surface markers. This has previously led to confusion between these two subsets. In addition, another PP DC subset, termed double-negative (DN), remains poorly characterized. Here we show that both DN and CD11b ${ }^{+} \mathrm{cDCs}$ belong to a unique SIRP $\alpha^{+} \mathrm{cDC}$ subset. At steady state, $\mathrm{CDCs}$ and TIM- $4^{+}$macrophages are mainly located in T-cell zones, i.e., interfollicular regions, whereas a majority of subepithelial phagocytes are monocyte-derived cells, namely, LysoDCs and TIM-4 ${ }^{-}$

macrophages. Finally, oral administration of a Toll-like receptor 7 ligand induces at least three TNF-dependent events: (i) migration of dome-associated villus CDCs in interfollicular regions, (ii) increase of CD $8 \alpha^{+}$interfollicular CDC number, and (iii) activation of both $\mathrm{CD} 11 \mathrm{~b}^{+}$and $\mathrm{CD} 8 \alpha^{+}$interfollicular CDCs. The latter is marked by a genetic reprograming leading to the upregulation of type I interferon-stimulated and of both immuno-stimulatory and -inhibitory gene expression.

\section{INTRODUCTION}

Among antigen-presenting cells, dendritic cells (DCs) are the most efficient at initiating antigen-specific responses, inducing differentiation of both naive $\mathrm{CD} 4{ }^{+}$and $\mathrm{CD} 8{ }^{+}{ }^{\mathrm{T}}$ cells. ${ }^{1} \mathrm{DCs}$ have a remarkable pattern of functional specialization overtime, also called maturation. It includes specific mechanisms to control antigen uptake, processing, and presentation. ${ }^{2,3}$ In their immature state, DCs detect and capture incoming pathogens. Then, upon stimulation, they begin a complex process of differentiation that involves a profound genetic reprograming. ${ }^{4,5}$ This leads to important phenotypic, morphological, and functional changes required for their migration to lymph node $\mathrm{T}$-cell zones as well as for antigen presentation and priming of naive $\mathrm{T}$ cells to mount an appropriate adaptive immune response.

To give rise to protective immunity, mucosal vaccines require the use of adjuvants that override the natural bias of the mucosal immune system toward the induction of tolerance. Knowing how these adjuvants are able to modify DC behavior in vivo is crucial to a better understanding of the mucosal immune response initiation, especially in primary inductive sites such as Peyer's patches (PPs) of the small intestine. Nevertheless, there is still little information about the alteration induced by adjuvants on PP DC populations. Cholera toxin is a powerful mucosal adjuvant that induces the migration of cells expressing CD11c, a marker of mouse gut DCs and macrophages, ${ }^{6}$ into the follicle-associated epithelium (FAE) followed by the migration of microsphere-loaded $\mathrm{CD} 11 \mathrm{c}^{+}$phagocytes in interfollicular regions (IFRs) enriched in T cells. ${ }^{7,8}$ Several Toll-like receptor (TLR) ligands also induce $\mathrm{CD} 11 \mathrm{c}^{+}$cell migration into the FAE. ${ }^{7,9-11}$ Finally, R848, a TLR7 agonist, induces an increase of $\mathrm{CD}_{103}{ }^{+}$cells, presumably DCs, in the IFR of rat PP. ${ }^{12}$ However, these $\mathrm{CD} 11 \mathrm{c}^{+}$or $\mathrm{CD} 103^{+}$cells have not been characterized further and could correspond to different subsets of DC or macrophages.

Mouse common DC precursor (CDP)-derived DCs, also termed conventional DC (cDCs), encompass indeed two major subsets that have been initially characterized by the expression

\footnotetext{
${ }^{1}$ Centre d Immunologie de Marseille-Luminy, CIML, Aix Marseille Univ, CNRS, INSERM, Marseille, France. Correspondence: H Lelouard (lelouard@ciml.univ-mrs.fr)

${ }^{2}$ These authors contributed equally to this work.

Received 18 July 2016; accepted 8 March 2017; published online 5 April 2017. doi:10.1038/mi.2017.30
} 
of either $\mathrm{CD} 8 \alpha(\mathrm{cDC} 1)$ or $\mathrm{CD} 11 \mathrm{~b}(\mathrm{cDC} 2)$, in addition to $\mathrm{CD} 11 \mathrm{c}$ and major histocompatibility complex class II (MHCII) ${ }^{13}$ $\mathrm{CD} 8 \alpha^{+}$cDCs have been well described in different tissues including PPs where they are located in the IFRs. ${ }^{14-16}$ Unlike $\mathrm{CD} 8 \alpha^{+}$cDCs, $\mathrm{CD} 11 \mathrm{~b}^{+}$cDCs remain poorly characterized depending on the examined tissue. This is mainly due to the overlap of their surface markers (i.e., CD11c, CD11b, and MHCII) with macrophages. ${ }^{6}$ Thus, we recently showed that PP $\mathrm{CD} 11 \mathrm{c}^{\text {hi }} \mathrm{CD} 11 \mathrm{~b}^{+}$mononuclear phagocytes comprise $\mathrm{CD} 11 \mathrm{~b}^{+}$ cDCs but also lysozyme-expressing DCs (LysoDCs) and macrophages (LysoMacs) that, unlike CD11 ${ }^{+} \mathrm{cDC}$, are both $\mathrm{CX}_{3} \mathrm{CR} 1^{+}$monocyte-derived cells. ${ }^{17}$ Unlike LysoDCs, LysoMacs display CD4 at their surface and encompass two subsets based on the expression of the apoptotic cell receptor TIM-4. TIM $-4^{+}$LysoMacs are mainly located in the T-cell zone of PP, i.e., the IFR, and in the lower part of the follicle, whereas TIM- $4^{-}$LysoMacs are located in the upper part of the follicle and in the subepithelial dome (SED). ${ }^{17}$ Particulate antigens and pathogenic bacteria that have been transported through M-cells of the FAE are mainly internalized in the SED by TIM- $4^{-}$ LysoMacs and LysoDCs, which both display strong innate antiviral and antibacterial gene signatures. ${ }^{17-19}$ In addition, LysoDC dendrites can directly sample luminal antigens through $\mathrm{M}$-cell-specific transcellular pores by a mechanism independent of $\mathrm{CX}_{3} \mathrm{CR} 1$ expression. ${ }^{17,18}$ LysoDC and LysoMac recruitment is independent of microbiota colonization. ${ }^{17}$ Finally, unlike LysoMacs, LysoDCs display a rapid renewal rate, strongly express genes of the MHCII presentation pathway and prime naive helper $\mathrm{T}$ cells for interferon- $\gamma$ production in vitro. ${ }^{17}$

If phenotypic distinction between PP CD11b ${ }^{+} \mathrm{cDCs}$ and monocyte-derived DCs has recently been solved, ${ }^{17}$ confusion still remains concerning their location and functions. Moreover, PP $\mathrm{CD}_{11} \mathrm{~b}^{+} \mathrm{cDCs}$ encompass both dome $\mathrm{cDCs}$ and dome-associated villus (DAV) cDCs. ${ }^{17}$ Finally, PPs contain another $\mathrm{CDC}$ subset termed double-negative DCs (DN DCs), as it neither expresses CD11b nor CD8 $\alpha^{14,15}$ Both CD11b ${ }^{+}$and DN cDCs express Clec $4 a 4$ and SIRP $\alpha$, whereas CD $8 \alpha^{+}$cDCs do not. ${ }^{17}$ Moreover, the transcription factor Batf3, which is required for the differentiation of $\mathrm{CD} 8 \alpha^{+} \mathrm{cDCs}$, is dispensable for $\mathrm{CD}_{11 \mathrm{~b}^{+}}$and DN cDCs. ${ }^{17}$ Finally, CD8 $\alpha^{+}$cDCs prime naive helper $\mathrm{T}$ cells to secrete interferon- $\gamma$, whereas $\mathrm{CD} 11 \mathrm{~b}^{+}$ and DN cDCs do not. ${ }^{17}$ Thus, DN DCs may be more closely related to $\mathrm{CD} 11 \mathrm{~b}^{+}$than to $\mathrm{CD} 8 \alpha^{+} \mathrm{cDCs}$.

Here we have studied into details the genetic relationship, the distribution, and location of the different dome $\mathrm{CDC}$ subsets and investigated their activation transcriptional profile upon oral delivery of R848 as a model of mucosal adjuvant. We also show that, upon stimulation, DAV cDCs migrate to the T-cell zone of PPs, i.e., the IFR.

\section{RESULTS}

\section{DN and CD11b ${ }^{+}$dome cDCs belong to a unique SIRP $\alpha^{+}$ cDC subset}

We established a flow cytometry gating strategy to sort CD $8 \alpha^{+}$, $\mathrm{DN}$, and $\mathrm{CD}_{11 \mathrm{~b}^{+}}$dome cDCs and study their genetic relationship. $\mathrm{CD} 11 \mathrm{c}^{\mathrm{hi}}$ monocyte-derived cells, i.e., LysoDCs and LysoMacs, express BST2, whereas cDCs do not. ${ }^{17}$ Among cDCs, DN and CD $11 b^{+}$cDCs express SIRP $\alpha$, whereas CD $8 \alpha^{+}$ cDCs do not. Thus, CD11c ${ }^{\text {hi }} \mathrm{MHCII}^{+}$cells were first gated into three populations based on the expression of BST2 and SIRP $\alpha$ : LysoDCs/LysoMacs (SIRP $\left.\alpha^{\text {hi }} \mathrm{BST}^{+}{ }^{+}\right), \mathrm{DN} / \mathrm{CD} 11 \mathrm{~b}^{+}$ cDCs $\left(\mathrm{SIRP} \alpha^{+} \mathrm{BST}^{-}\right)$, and $\mathrm{CD} 8 \alpha^{+}$cDCs $\left(\mathrm{SIRP}^{-}{ }^{-} \mathrm{BST} 2^{-}\right.$; Figure 1a). Then, SIRP $\alpha^{+}$BST2 $^{-}$cells were further separated into three subsets based on CD11b differential expression: ${ }^{17}$ dome DN $\left(\mathrm{CD} 11 b^{-}\right)$, dome $\mathrm{CD} 11 \mathrm{~b}^{+}\left(\mathrm{CD} 11 \mathrm{~b}^{\text {int }}\right)$, and $\mathrm{DAV}$ $\mathrm{CD}_{11} \mathrm{~b}^{+}\left(\mathrm{CD} 11 \mathrm{~b}^{\mathrm{hi}}\right) \mathrm{cDCs}$ (Figure 1a). In addition, LysoMacs were separated into TIM- $4^{-}$and TIM- $4^{+}$subsets. Triplicates of TIM- $4^{-}$and TIM- $4^{+}$LysoMacs, CD $8 \alpha^{+}$and DN dome $\mathrm{cDCs}$, and quintuplicate of $\mathrm{CD} 11 \mathrm{~b}^{+}$dome $\mathrm{cDCs}$ were analyzed by mouse whole-genome microarray. Generated data were combined with those of LysoDCs and CD11b ${ }^{+}$dome cDCs previously obtained, ${ }^{17}$ and the transcriptional proximity between $\mathrm{CD} 11 \mathrm{c}^{\text {hi }}$ phagocytes was determined by hierarchical clustering (Figure 1b). Two main clusters were observed: one composed by cDCs and the other by monocyte-derived cells. Among the latter, LysoDCs clustered apart from TIM- $4^{-}$and TIM- ${ }^{+}$LysoMacs, whereas among cDCs, DN and CD11b ${ }^{+}$ cDCs clustered together apart from $\mathrm{CD} 8 \alpha^{+}$cDCs. We also performed a principal component analysis. The first principal component separated cells according to their origin, i.e., monocyte vs. CDP-derived cells (Figure 1c). Genes contributing to this axis included typical CDC markers (e.g., Itgae, Flt3, Btla, Id2, and Ccr7; Supplementary Table S1 online) on one side, and monocyte and macrophage markers on the other (e.g., Mertk, Mafb, Tcfec, Cx3cr1; Supplementary Table S1). The second principal component separated mainly SIRP $\alpha^{+}$(DN and CD $11 b^{+}$cDCs, LysoDCs, and LysoMacs) from SIRP $\alpha^{-}\left(\mathrm{CD} 8 \alpha^{+}\right.$cDCs $)$phagocytes and contributing genes were either associated with the $\mathrm{CD} 8 \alpha^{+} \mathrm{cDC}$ subset (e.g., Clec9a, Xcr1, Tlr3, Tlr11, Cd8a, Cadm1, and Irf8; Supplementary Table S1) or the $\mathrm{CD}_{11 b^{+}} \mathrm{cDC}$ subset (e.g., Clec4a4, Sirpa, Sirpb1, Csf1r, and Il22ra2; Supplementary Table S1). Principal component analysis failed to discriminate DN from $\mathrm{CD}_{11} \mathrm{~b}^{+}$cDCs confirming their close genetic relatedness (Figure 1c). In line with these results, the genes encoding the key transcriptional factors IRF4 and IRF8 involved either in $\mathrm{CD} 11 \mathrm{~b}^{+}$or $\mathrm{CD} 8 \mathrm{a}^{+} \mathrm{cDC}$ subset commitment were either expressed or not by DN cDCs, respectively (Supplementary Figure S1A). Only 230 differentially expressed genes (DEGs) were found between $\mathrm{DN}$ and $\mathrm{CD} 11 \mathrm{~b}^{+} \mathrm{cDCs}$, whereas 1,098 DEGs distinguished the latter from CD $8 \alpha^{+}$cDCs. The top 20 of $\mathrm{CD} 11 \mathrm{~b}^{+}$vs. DN cDC upregulated genes comprised typical DC maturation transcripts encoding molecules involved in migration to T-cell zones (lymph node homing chemokine receptor CCR7) and in attraction (chemokines CCL17 and CCL22) and stimulation (the cystine/glutamate antiporter SLC7A11 and the proinflammatory cytokine interleukin-6) of effector cells (Supplementary Figure S1B). In addition, the genes encoding for fascin1 ( Fscn1), which is an actin-bundling protein involved in DC migration upon maturation, ${ }^{20}$ and for the transcription factor STAT4, which is induced upon DC maturation, ${ }^{21}$ were also upregulated in $\mathrm{CD} 11 \mathrm{~b}^{+} \mathrm{cDCs}$ 
(Supplementary Figure S1A). Thus, DN and CD11b ${ }^{+}$dome cDCs were likely different homeostatic maturation stages of PP SIRP $\alpha^{+}$cDCs. To strengthen this hypothesis, we performed a multiple gene set enrichment analysis using the recently developed BubbleGUM software. ${ }^{22}$ Gene signatures were obtained from mouse and cross-species studies performed by several laboratories (Supplementary Table S2). ${ }^{4,523-29}$ First, we corroborated the monocytic and CDP origin of LysoDCs/ LysoMacs and DN/CD $11 b^{+} / \mathrm{CD} 8 \alpha^{+}$DCs, respectively (Figure 1d, pink boxes). Second, we confirmed that both LysoDCs and $\mathrm{CD}_{11} \mathrm{~b}^{+}$cDCs were enriched for a CD11b cDC2 cDC gene signature as compared to LysoMacs, whereas a
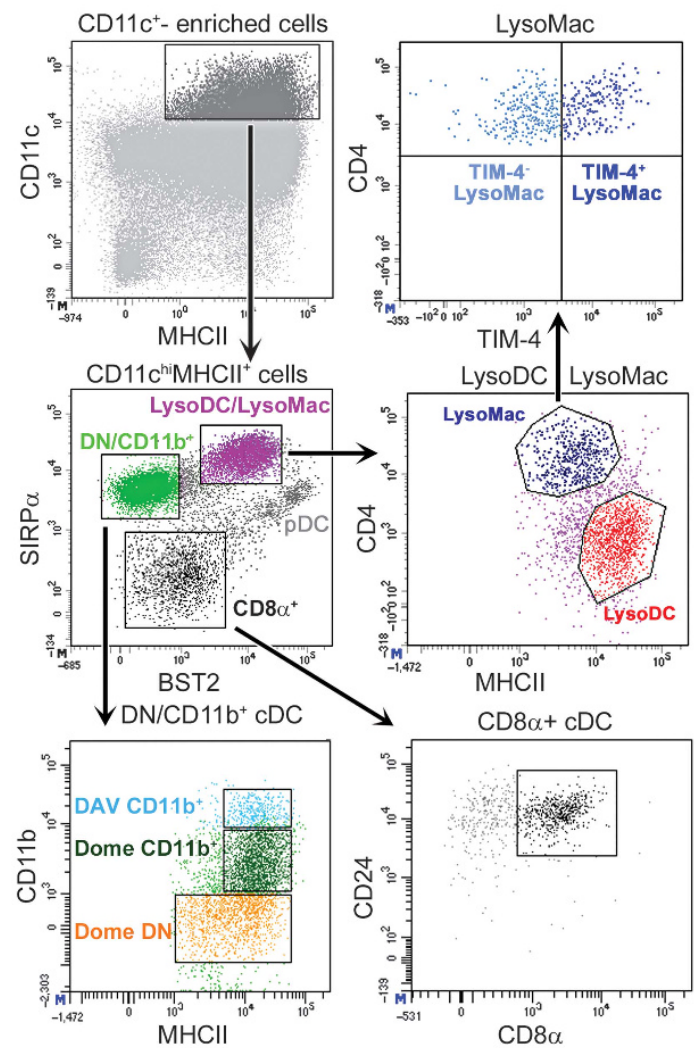

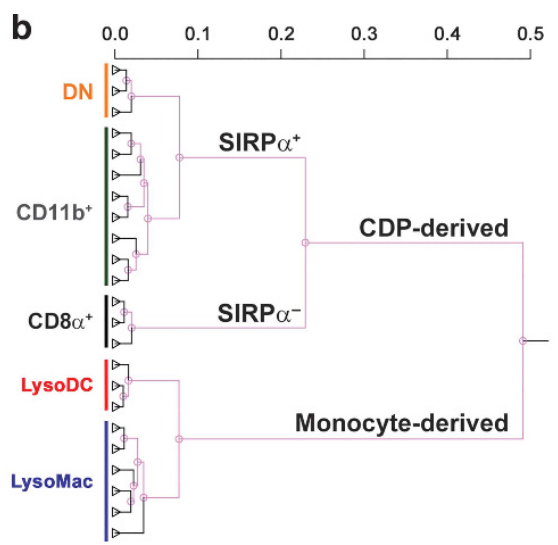

c

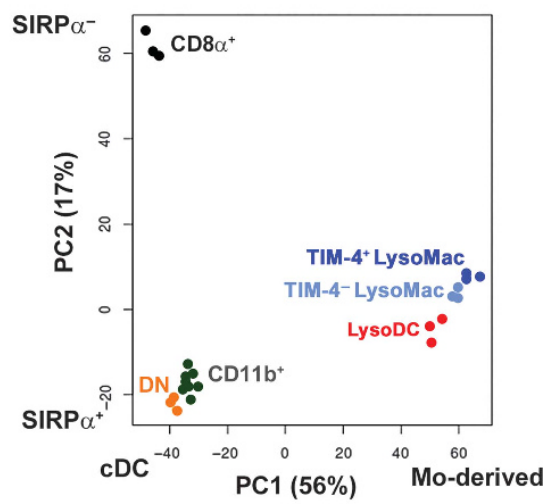

d

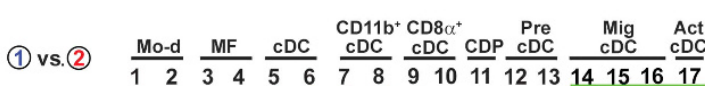
DN vs.CD11 $\mathrm{b}^{+} \bigcirc 00000000$ DN vs. CD8 $\alpha^{+} \bigcirc 00000000$

DN vs. LysoDC 00000000000000000 DN vs. LysoMac1 100000000000

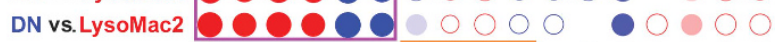
CD11 b+ vs. CD8 $\alpha^{+} 0000000000000000$ CD11 b+ vs. LysoDC 0000000000000000 CD11b+vs.LysoMac1 0000000000000000 CD11b+vs. LysoMac2 000000000000000

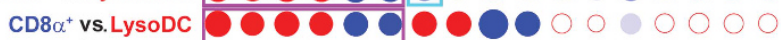
CD8 $\alpha^{+}$vs. LysoMac1 10000000000000000 CD8 $\alpha^{+}$vs. LysoMac2 00000000000000000 LysoDC vs. LysoMac1 $0000000 \bigcirc 0000 \bigcirc 000$ LysoDC vs. LysoMac2 0000000000000000

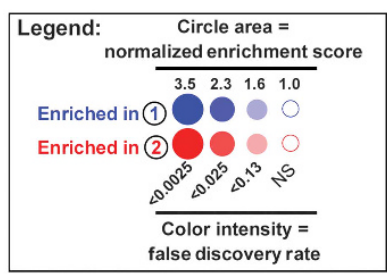

$1,3,5,7,9,11-17:$ mouse signatures 2, 4, 6, 8, 10: cross-species signatures 1, 2, 8, 10: Vu Manh et al., 2015 3: Gautier et al., 2012 4, 6: Xue et al., 2014 5, 15: Miller et al., 2012

7, 9, 14: generated from Immgen database (Heng et al., 2008) 11, 12, 13: Grajales-Reyes et al., 2015 16: Tamoutounour et al., 2013 7: Manh et al., 2013
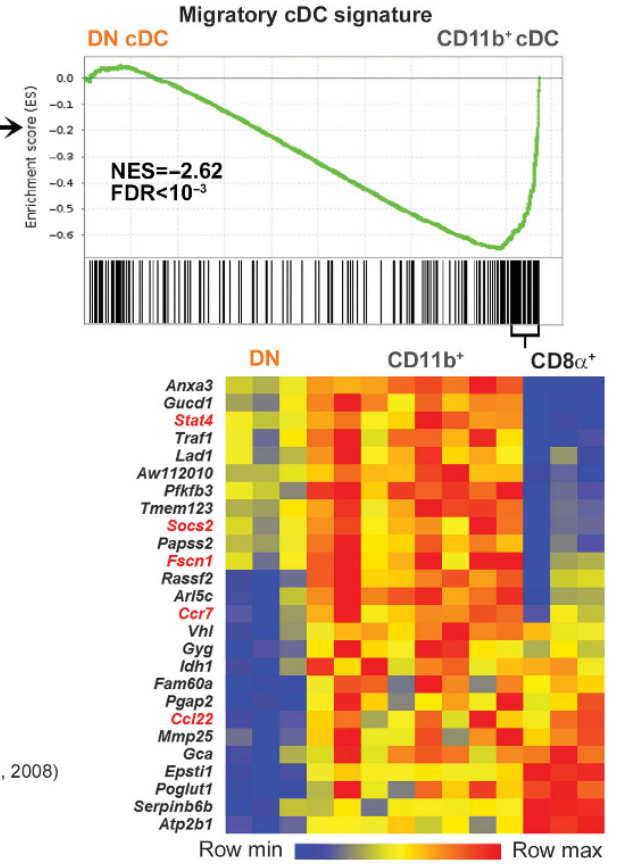
there was a lack of enrichment for this signature between $\mathrm{CD}_{11 \mathrm{~b}^{+}} \mathrm{cDCs}$ and LysoDCs (Figure 1d, blue boxes). Third, we confirmed that both $\mathrm{DN}$ and $\mathrm{CD}_{11 \mathrm{~b}^{+}}$belonged to the $\mathrm{CD}_{11 \mathrm{~b}^{+} / \mathrm{cDC} 2}$ lineage (Figure 1d, orange boxes). Finally, BubbleGUM analysis supported that DN and CD11b ${ }^{+} \mathrm{cDCs}$ differed by their maturation state as underlined by the enrichment of the latter for migratory/activated CDC gene signatures (Figure 1d, green box). The strongest enrichment score was obtained for the migratory $\mathrm{CDC}$ signature, which includes key DC maturation genes such as Stat4, Socs2, Fscn1, $C c r 7$, and Ccl22 (Figure 1d, right panel). These data establish a close genetic relationship between $\mathrm{DN}$ and $\mathrm{CD} 11 \mathrm{~b}^{+} \mathrm{cDCs}$, and suggest that DN cDCs likely represent an immature stage of $\mathrm{CD}_{11 \mathrm{~b}}{ }^{+} \mathrm{cDCs}$.

\section{SIRPa $\alpha^{+}$dome $\mathrm{cDCs}$ form a continuum of cells ranging from CD11c ${ }^{\text {hi }}$ SIRP $\alpha^{+}$BST2- ${ }^{-}$D11b ${ }^{-}$MHCII $^{\text {lo }}$ CD24 $^{\text {hi }}$ EpCAM $^{\text {lo }}$ JAM-A $^{\text {int }}$ to CD11c ${ }^{\text {hi }}$ SIRP $\alpha^{+}$BST2 ${ }^{-}$CD11b $^{\text {int }}$ MHCI $^{\text {hi }}$ CD24 $^{\text {int }}$ EpCAM $^{\text {int }} J A M-A^{\text {hi }}$}

A hallmark of DC maturation is the increased surface display of MHCII molecules for antigen presentation. ${ }^{3}$ Interestingly, based on MHCII and CD11b expression, SIRP $\alpha^{+}$dome cDCs formed a continuum of cells ranging from $\mathrm{CD}_{11} \mathrm{~b}^{-} \mathrm{MHCII}{ }^{\mathrm{lo}}$ to $\mathrm{CD}_{11} \mathrm{~b}^{\text {int }} \mathrm{MHCII}^{\mathrm{hi}}$ (Figure 1a and 2a). This suggests that transitional states exist between DN $\left(\mathrm{CD} 11 \mathrm{~b}^{-} \mathrm{MHCII}^{\mathrm{lo}}\right)$ and $\mathrm{CD}_{11 \mathrm{~b}}{ }^{+}\left(\mathrm{CD} 11 \mathrm{~b}^{\text {int }} \mathrm{MHCII}^{\mathrm{hi}}\right)$ cDCs. In order to study these transitional states, we divided SIRP $\alpha^{+}$cDCs into four putative differentiation stages (Figure 2a). We selected three cell surface markers, namely, EpCAM, JAM-A, and CD24, based on differential gene expression between $\mathrm{DN}$ and $\mathrm{CD} 11 \mathrm{~b}^{+} \mathrm{cDCs}$ and antibody availability (Figure 2b). We observed a progressive increase of CD11b, MHCII, EPCAM, and JAM-A surface expression from the first (I, CD11 b ${ }^{-} \mathrm{MHCII}^{\mathrm{lo}}$ cells, $\mathrm{DN}$ cDCs) to the last cell population (IV, CD11 $\mathrm{b}^{\text {int }} \mathrm{MHCII}{ }^{\text {hi }}$ cells, CD11b ${ }^{+}$ cDCs), whereas CD24 expression decreased along the same path (Figure 2c). To assess whether DN cDCs could indeed express
CD11b, we checked the phenotype of isolated DN cDCs after an overnight culture. Both DN and CD11b ${ }^{+} \mathrm{cDCs}$ increased their surface levels of MHCII after culture and as expected, nearly half of DN cDCs acquired surface expression of CD11b (Figure 2d). We also observed that DN cDCs incorporated the marker of proliferation EdU slightly but significantly faster than $\mathrm{CD} 11 \mathrm{~b}^{+}$ cDCs in vivo, suggesting that they were the first to be replaced by progenitors (Figure 2e; Supplementary Figure S1C).

Altogether, these results suggest that $\mathrm{DN}$ and $\mathrm{CD} 11 \mathrm{~b}^{+} \mathrm{cDCs}$ likely represent the two extremities of a PP SIRP $\alpha^{+}$cDC developmental path (see Figure 2f for a differentiation model of $\mathrm{SIRP}^{+}$dome cDCs).

\section{CD11c ${ }^{\text {hi }} C D 11 b^{\text {hi }}$ cells of the SED are mainly LysoDCs and LysoMacs}

Unlike Ccr7, the gene encoding CCR6, which is a chemokine receptor expressed by immature DCs ${ }^{30}$ was expressed at higher levels in DN than in CD11b ${ }^{+}$cDCs (Supplementary Figure S1A). CCL20, the ligand of CCR6, is secreted by the FAE in the SED, ${ }^{14,31}$ whereas CCL19 and CCL21, the ligands of CCR7, are expressed in the IFR. ${ }^{32-34}$ In agreement with these chemokine locations, CCR6 messenger RNA has been detected throughout the follicle and the SED but not in the IFR, whereas CCR7 messenger RNA expression has only been reported in the IFR. ${ }^{14}$ This suggests that $\mathrm{DN}$ and $\mathrm{CD} 11 \mathrm{~b}^{+} \mathrm{cDCs}$ could be attracted to the SED and the IFR, respectively. Nevertheless, CD11c ${ }^{+}$ $\mathrm{CD}_{11} \mathrm{~b}^{+}$cells are known to be mainly located in the SED but not in the IFR. ${ }^{14,19,31}$ However, when we examined into more details the phenotype of these subepithelial CD11 ${ }^{+} \mathrm{CD}_{11} \mathrm{~b}^{+}$ cells, most of them also expressed lysozyme and $\mathrm{CX}_{3} \mathrm{CR} 1$, indicating that they were LysoDCs or TIM- $4^{-}$LysoMacs but not $\mathrm{CD}_{11 \mathrm{~b}^{+}} \mathrm{cDCs}$ (Figure 3a). In addition and in agreement with previous reports, ${ }^{14,19,31}$ we only detected rare $\mathrm{CD} 11 \mathrm{c}^{+}$ $\mathrm{CD} 1 \mathrm{~b}^{+}$cells in the IFR (Figure $\mathbf{3 b}$ ). However, the relative ratio of $\mathrm{PP}$ mononuclear phagocyte subsets obtained after tissue dissociation of C57BL/6 mouse PP indicated that $\mathrm{CD} 11 \mathrm{~b}^{+}$

Figure 1 Phenotypic and transcriptional profiles of PP phagocytes. (a) Gating strategy for LysoDCs, TIM-4 ${ }^{-}$and TIM-4 ${ }^{+}$LysoMacs, and CD8 $\alpha^{+}$ CD11b ${ }^{+}$, and DN dome cDCs sorting is shown. CD11 $\mathrm{c}^{\mathrm{hi}} \mathrm{MHCll}{ }^{+}$cells were selected among PP CD11c ${ }^{+}$-enriched cells and analysed for SIRP $\alpha$ and BST2 expression. Monocyte-derived cells (LysoDCs and LysoMacs) were selected as SIRP $\alpha^{\text {hi BST2 }}{ }^{+}$cells. Then, LysoMacs and LysoDCs were separated using their CD4 and MHCll differential expression before the splitting of LysoMacs in two subsets according to their TIM-4 expression. Continuing with BST2 ${ }^{-}$cells, CD8 $\alpha^{+}$cDCs were identified as SIRP $\alpha^{-}$cells and their identity was further confirmed using CD8a and CD24 staining. Finally, surface expression of CD11b on SIRP $\alpha^{+} \mathrm{BST}^{-}$cells allowed to distinguish DN and CD11 $\mathrm{b}^{+}$dome cDCs from dome-associated villus cDCs. (b-d) Gene expression analysis of PP phagocyte subsets obtained from three to five independent cell-sorting experiments with pooled cells from 42 mice per experiment combined with our previous gene array data deposited into Gene Expression Omnibus repository under accession number GSE65514. See also Supplementary Figure S1A,B. (b) Hierarchical clustering of PP phagocytes, by Pearson correlation distance and Ward's aggregation, after selection of genes with a differential expression between any subset $>1.5$-fold (5,751 probes).(c) Principal component analysis of PP phagocytes. See also Supplementary Table S1. (d) Multiple gene set enrichment analysis of PP phagocytes. Left panel: BubbleGUM software was used to compare the expression of different gene signatures between each phagocyte subset. As shown in the lower left legend, gene set enrichment in one subset is shown by a bubble of the subset color. The bubble size and the color darkness are related to the normalized enrichment score and to the false discovery rate, respectively. Thus, stronger and more significant enrichments are represented by bigger and darker bubbles. Pink boxes highlight monocyte-derived vs. cDC gene signature enrichment between LysoDCs or lysoMacs and DN, CD11b ${ }^{+}$, or CD8 $\alpha^{+}$cDCs. Blue boxes highlight the enrichment or lack of enrichment for $\mathrm{cDC}$ and $\mathrm{CD} 11 \mathrm{~b}^{+} \mathrm{cDC}$ gene signatures in LysoDCs as compared to LysoMacs or in CD11 ${ }^{+} \mathrm{cDCs}$ as compared to LysoDCs or LysoMacs. Orange boxes show enrichment of the CD11b ${ }^{+}$vs. CD $8 \alpha^{+} \mathrm{cDC}$ gene signatures between DN or CD $11 b^{+} \mathrm{cDCs}$ and CD8 $\alpha^{+} \mathrm{cDCs}$. Green box highlights the migratory/activated $\mathrm{CDC}$ gene signature of $\mathrm{CD} 11 \mathrm{~b}^{+} \mathrm{cDCs}$ as compared to DN cDCs. Right panel: an enrichment plot for the migratory $D C$ gene signature that best distinguished $D N$ and $C D 11 b^{+} \mathrm{cDCs}$ (top) and a heatmap of the genes from the signature that contribute the most to the enrichment score (bottom) are shown. Genes with well-defined functions in migratory cDCs are in red. See Supplementary Table S2 for the list of gene signatures. Act, activated; $C D C$, conventional dendritic cell; CDP, common DC precursor; DN, double negative; FDR, false discovery rate; LysoDC, lysozyme-expressing dendritic cell; LysoMac, lysozyme-expressing macrophage; LysoMac1, TIM-4- ${ }^{-}$LysoMac; LysoMac2, TIM-4 ${ }^{+}$LysoMac; MF, macrophage; Mig, migratory; Mo-d, monocyte-derived; NES, normalized enrichment score; PP, Peyer's patch. 
a
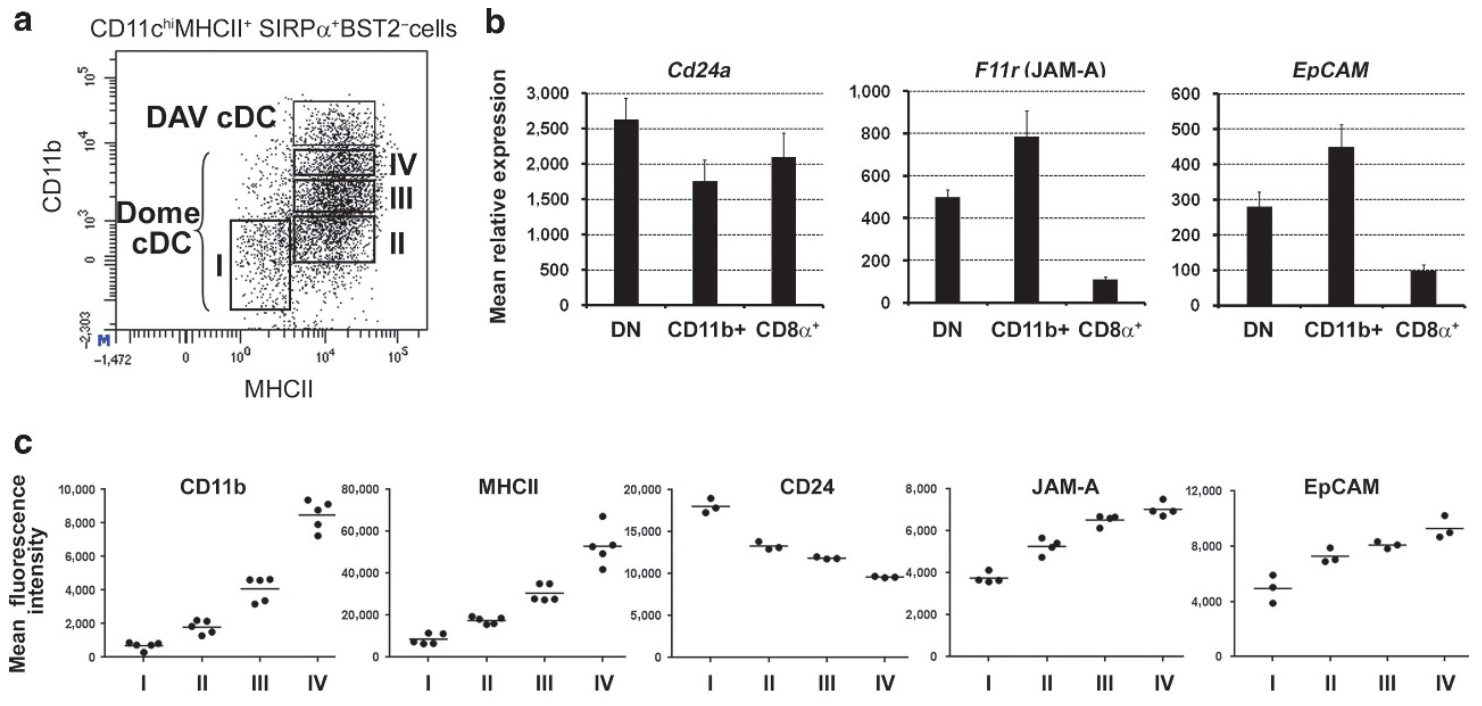

d
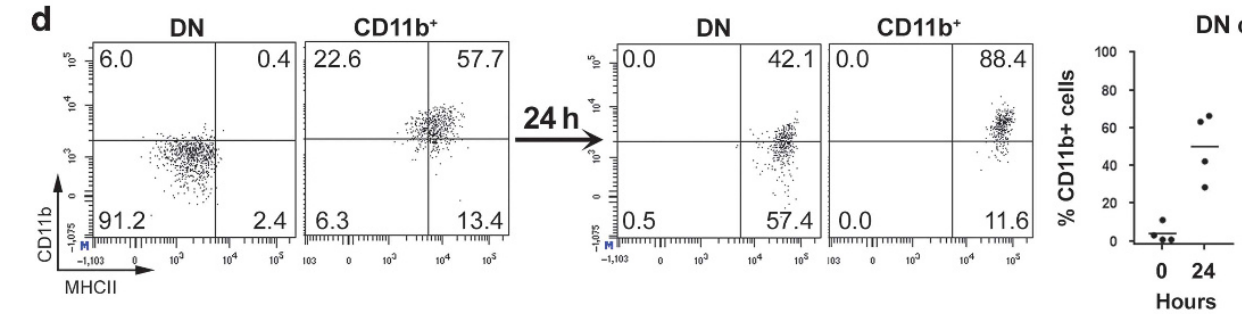

DN cDC subset

e

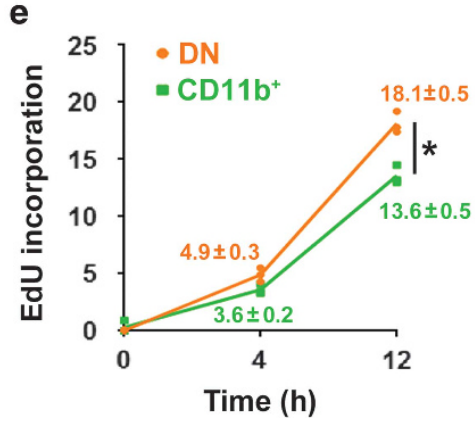

f
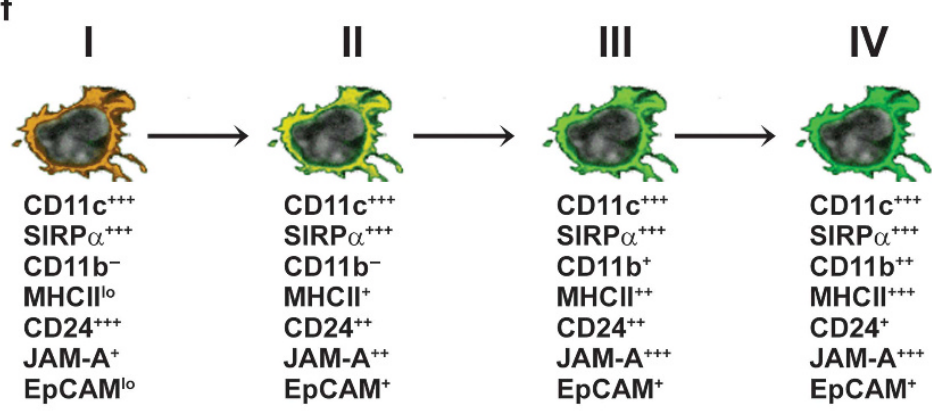

Figure 2 Model of SIRP $\alpha^{+}$dome cDCs developmental pathway. (a) Identification of four putative developmental stages of SIRP $\alpha^{+}$cDCs based on CD11b and MHCII surface expression. Stage I, CD11b ${ }^{-}$MHCII $^{\text {; }}$; stage II, CD11b ${ }^{-} \mathrm{MHCII}^{\text {int; }}$; stage III, CD11 ${ }^{\text {lo }} \mathrm{MHCII}^{\text {hi }}$; and stage IV,

$\mathrm{CD}_{11} \mathrm{~b}^{\text {int }} \mathrm{MHClI}^{\mathrm{hi}}$. (b) Normalized mean relative expression \pm s.d. of $C d 24, F 11$, and Epcam in dome cDC subsets. (c) Mean fluorescence intensity of CD11b, MHCII, CD24, JAM-A, and EpCAM in the subpopulations of SIRP $\alpha^{+}$cDCs defined in $\mathbf{a}$. Three to five independent experiments are shown. (d) Alteration of $\mathrm{DN}$ and $\mathrm{CD} 11 \mathrm{~b}{ }^{+} \mathrm{CDC}$ subset phenotype upon $24 \mathrm{~h}$ in vitro culture. Four left dot plots: representative surface expression of $\mathrm{CD} 11 \mathrm{~b}$ and $\mathrm{MHCll}$ in isolated live DN and CD11 ${ }^{+}$dome cDCs before and after culture. Survival rate after $24 \mathrm{~h}$ culture varied between 10 and $30 \%$ in both subsets depending on the experiment. The last two plots are a summary of four independent experiments showing the percentage of CD11b ${ }^{+}$cells and CD11b mean fluorescence intensity among live DN cDCs before and after culture. (e) Kinetics of EdU incorporation into DN and CD11b ${ }^{+}$cDCs. PPs were collected 0,4 , and $12 \mathrm{~h}$ after EdU administration to mice. ${ }^{*} P<0.05$; unpaired $T$-test with Welch's correction performed from data of three independent experiments. See also Supplementary Figure S1C. (f) Model of SIRP $\alpha^{+}$dome $c D C$ developmental pathway from DN to CD11b ${ }^{+} \mathrm{CDC}$. $\mathrm{CDC}$, conventional dendritic cell; DAV, dome-associated villus; DN, double negative; PP, Peyer's patch.

cDCs were the main subset (Figure 3c). Thus, we were unable to detect the main dome cDC subset by microscopy using CD11b as a marker. We also observed that $\mathrm{CX}_{3} \mathrm{CR} 1^{+}$cells of the IFR were not stained with $\mathrm{CD} 11 \mathrm{~b}$ (Figure $3 \mathbf{b}$ ), indicating that the expression of CD11b at the surface of TIM- $4^{+}$LysoMacs, which represent most $\mathrm{CX}_{3} \mathrm{CR} 1^{+}$cells of the IFR (Figure 3d), was too low to be detected by microscopy. Altogether, these data suggest that some phagocytes positive for CD11b by flow cytometry may not be detectable by microscopy with this marker. LysoDCs and CD11b ${ }^{+}$DAV cDCs expressed indeed more CD11b than TIM- $4^{+}$LysoMacs and CD11b ${ }^{+}$ dome cDCs by flow cytometry (Figure 3e; Supplementary Figure S2A). Thus, we established a flow cytometry-correlated minimal threshold of detection of the marker CD11b by microscopy (Figure 3e; Supplementary Figure S2A). In agreement with this threshold, LysoDCs, $\mathrm{CD} 11 \mathrm{c}^{+} \mathrm{CX}_{3} \mathrm{CR} 1^{-}$ DAV cDCs, $\mathrm{CD} 11 \mathrm{c}^{+} \mathrm{CX}_{3} \mathrm{CR} 1^{+}$DAV macrophages, and some but not all TIM- $4^{-}$LysoMacs were stained for CD11b 

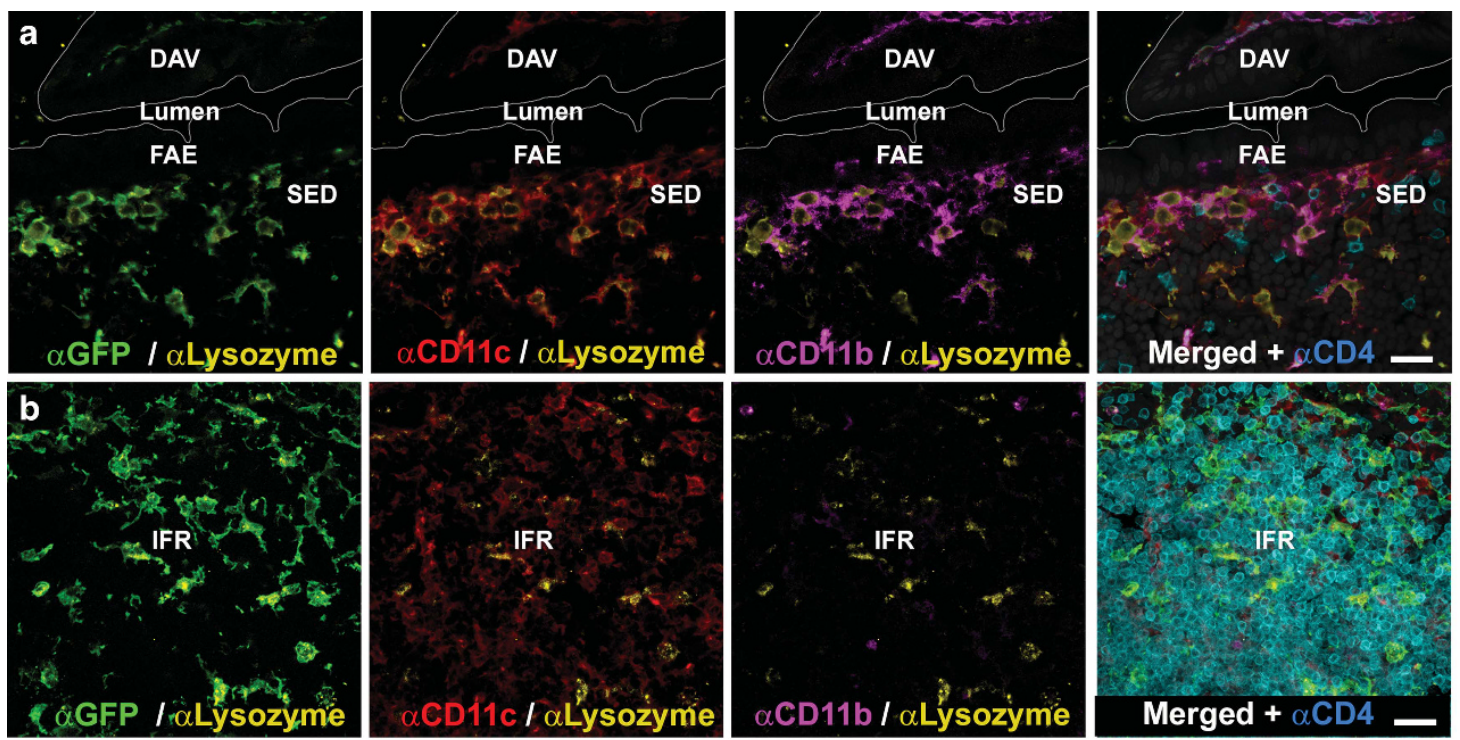

C
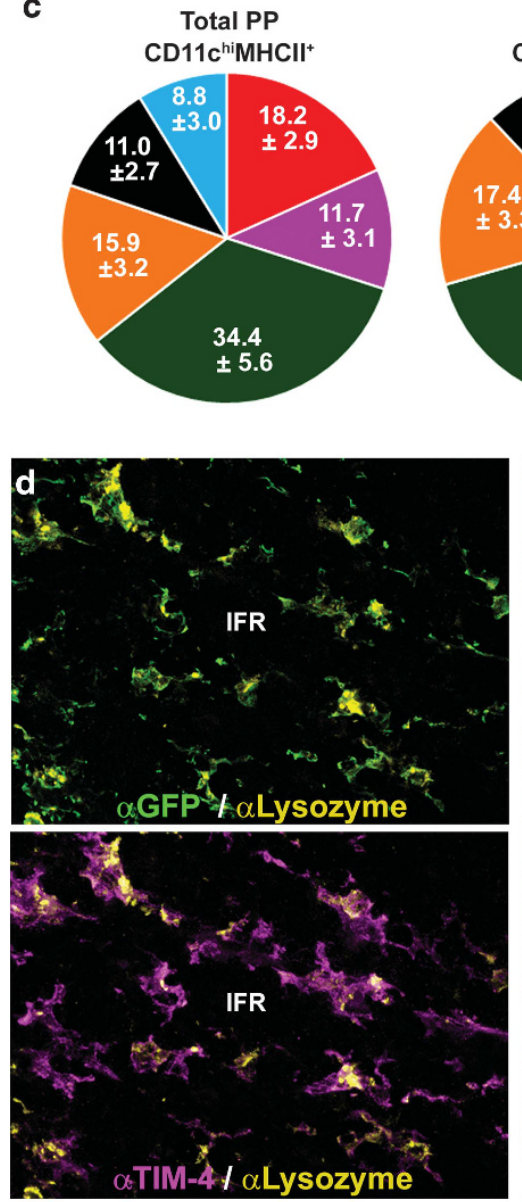

Dome

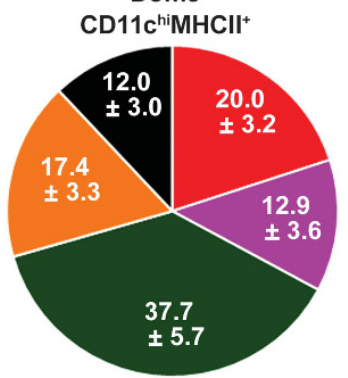

- DAV CD11b ${ }^{+} \mathrm{cDC}$

- LysoDC

- LysoMac

- Dome CD11b+cDC

- Dome DN cDC

- Dome $\mathrm{CD} 8 \alpha^{+} \mathrm{cDC}$

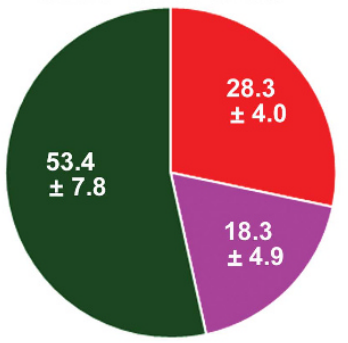

e $\quad$ D11 $\mathrm{c}^{\text {hi }} M \mathrm{MHCII}^{+} \mathrm{SIRP} \alpha^{\text {hi }} \mathrm{BST}^{+}$cells
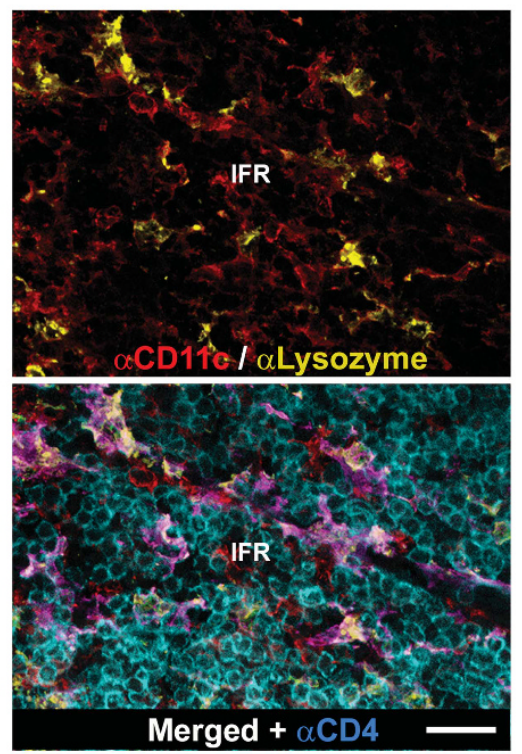

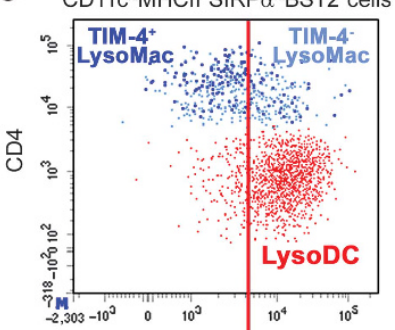

CD11 $\mathrm{c}^{\text {hi }} \mathrm{MHCII}{ }^{+}$SIRP $\alpha^{+} B S T 2^{-}$cells

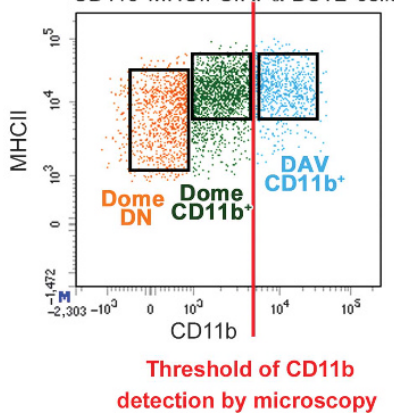

Figure 3 Distribution of PP phagocyte subsets at steady state. (a-b) Phenotype and location of CD11b ${ }^{+}$cells in PP. Confocal microscopy projection of $C x_{3} \mathrm{Cr}_{1-G F P}{ }^{-1+}$ mouse PP sections stained for GFP (green), CD11c (red), lysozyme (yellow), CD4 (cyan), and CD11b (magenta). (a) In the SED, most $\mathrm{CD} 11 \mathrm{~b}^{+}$cells were LysoDCs and LysoMacs $\left(\mathrm{CD} 11 \mathrm{c}^{+} \mathrm{CX}_{3} \mathrm{CR} 1^{+}\right.$lysozyme ${ }^{+}$cells). (b) In the IFR, CD11 ${ }^{+}$cells were either rare or absent. Bars, $20 \mu \mathrm{m}$. (c) Ratio of phagocyte subpopulations extracted from C57BL/6 mice PP (mean percentage \pm s.d. of 14 independent experiments). (d) Confocal microscopy projection of a $\mathrm{Cx}_{3} \mathrm{Cr1}$-GFP ${ }^{-/+}$mouse IFR stained for GFP (green), CD11c (red), lysozyme (yellow), CD4 (cyan), and TIM-4 (magenta). Most $\mathrm{CX}_{3} \mathrm{CR} 1{ }^{+} \mathrm{CD} 11 \mathrm{c}^{+}$lysozyme ${ }^{+}$cells of the IFR were TIM-4 $4^{+}$LysoMacs. Bar, $20 \mu \mathrm{m}$. (e) According to its expression levels, CD11b may or may not be detectable by confocal microscopy (red line = threshold of detection). See also Supplementary Figure S2. Results are representative of six independent experiments. DAV, dome-associated villus; FAE, follicle-associated epithelium; GFP, green fluorescent protein; IFR, interfollicular region; LysoDC, lysozyme-expressing dendritic cell; LysoMac, lysozyme-expressing macrophage; PP, Peyer's patch; SED, subepithelial dome. 
a

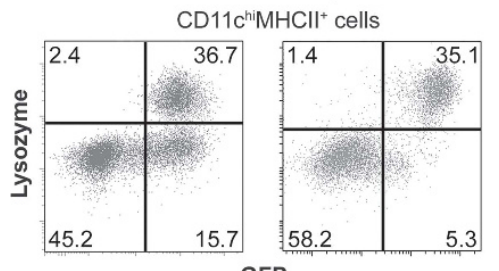

GFP
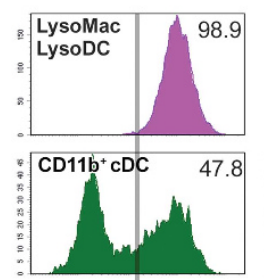

\begin{tabular}{|l|l|}
\hline DN cDC & 10.9 \\
\hline
\end{tabular}
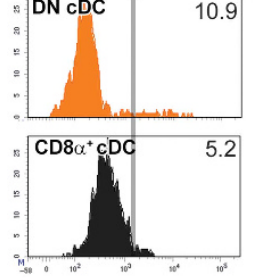

d

GFP b

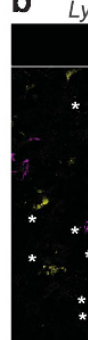

ys-EGFP mice

$\mathrm{Cx}_{3} \mathrm{Cr} 1-\mathrm{GFP}$ mice

C Lys-EGFP mice
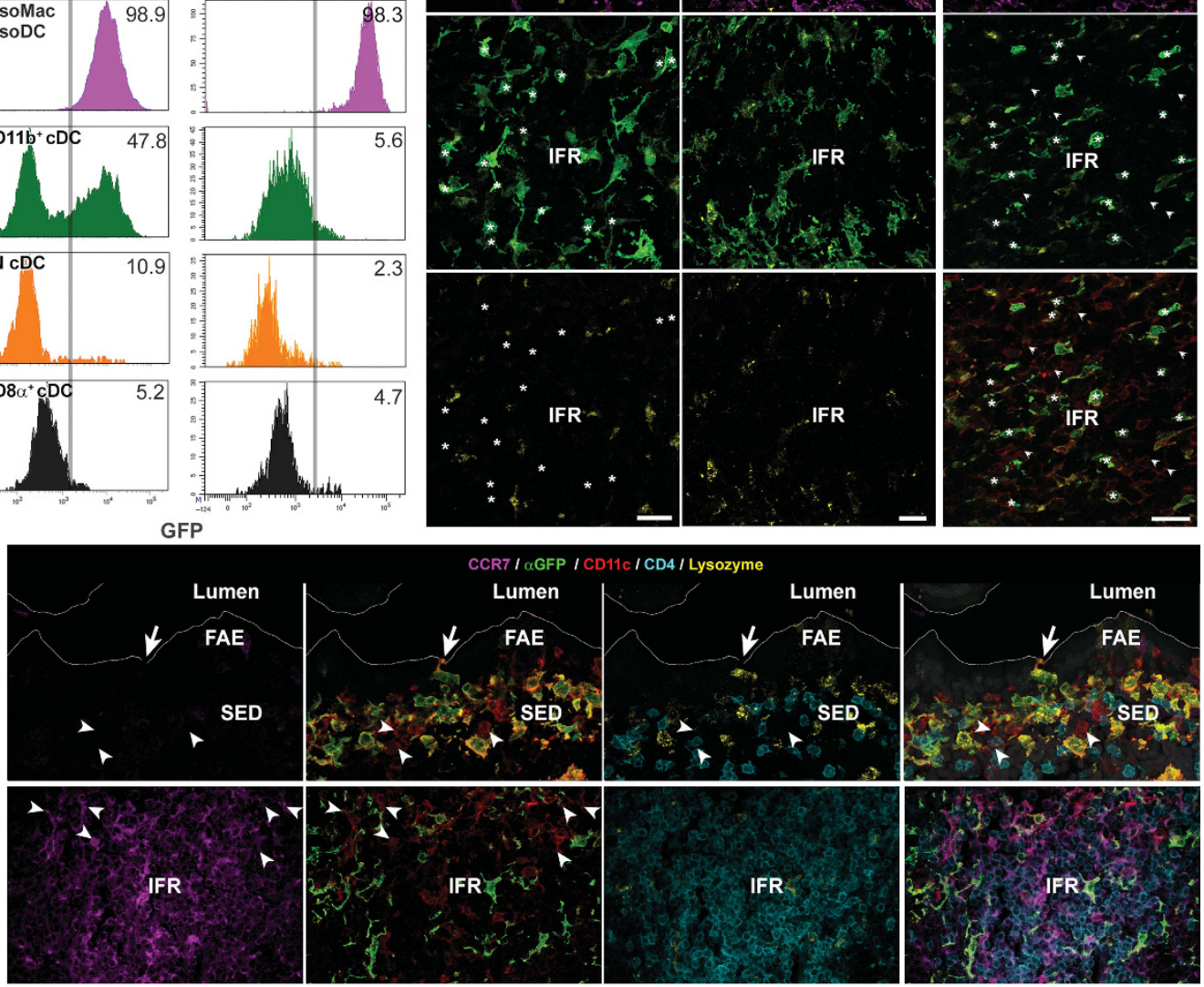

e
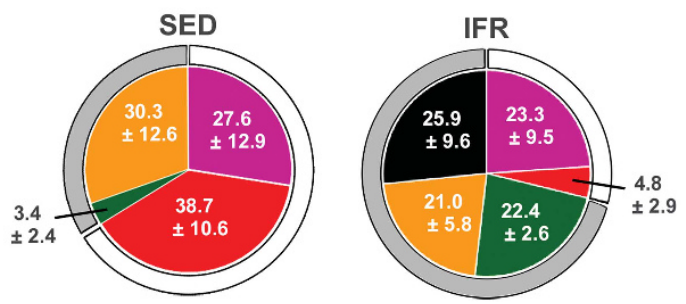

Figure 4 Location of PP CD11 $\mathrm{b}^{+} \mathrm{CDC}$ at steady state. (a) Expression of lysozyme and GFP in CD11 $\mathrm{c}^{\text {hi } M H C l I^{+}}$cells of PP from either lys-EGFP ${ }^{-1+}$ mice (left) or $\mathrm{Cx}_{3} \mathrm{Cr} 1-\mathrm{GFP} \mathrm{P}^{-1+}$ mice (right). GFP was only expressed by lysozyme-expressing cells in $\mathrm{Cx}_{3} C r 1-G F P^{-1+}$ mice, whereas it was also displayed by nearly half of CD11 $\mathrm{b}^{+} \mathrm{CDCs}$ in lys-EGFP mice. (b) Confocal microscopy projection of lys-EGFP ${ }^{-1+}$ (left) or $C x_{3} C r 1-G F P^{-1+}$ (right) mouse IFR stained for GFP (green), lysozyme (yellow), and TIM-4 (magenta). GFP was mainly expressed by TIM-4 $\left.{ }^{+}{\text {LysoMacs in } C x_{3} C r 1-G F P}^{-}\right)_{+}$mice, whereas it was also expressed by TIM- $4^{-}$lysozyme ${ }^{-}$cells $\left(C D 11 b^{+}{ }^{c D C}\right.$ subset) in lys-EGFP mice (asterisks). Bars, $20 \mu \mathrm{m}$. (c) Confocal microscopy projection of a lys-EGFP ${ }^{-1+}$ mouse IFR stained for GFP (green), CD11c (red), lysozyme (yellow), and SIRP $\alpha$ (magenta). In addition to lysozymeexpressing cells, SIRP $\alpha$ was expressed by CD11c ${ }^{+}$cells that displayed GFP (asterisks) or not (arrowheads). Bar, $20 \mu \mathrm{m}$. (d) Confocal microscopy projection of $\mathrm{Cx}_{3} \mathrm{Cr}_{1-G F P}{ }^{-1+}$ mouse SED (top) and IFR (bottom) stained for GFP (green), lysozyme (yellow), CD11c (red), CD4 (cyan), and CCR7 (magenta). CCR7 was not expressed by CDCs (CD11c ${ }^{+}$GFP $^{-}$Lysozyme $^{-}$, arrowheads) of the SED, whereas it was expressed by those of the IFR. Arrow points to a LysoDCs $\left(\mathrm{CD} 11 \mathrm{c}^{+} \mathrm{GFP}^{+} \mathrm{Lysozyme}^{+} \mathrm{CD}^{-}\right)$located in the FAE that sample luminal antigens by extending a dendrite into the lumen. See also Supplementary Figure S3. (e) Confocal microscopy assessment of the phagocyte subset ratio in the SED (left) and in the IFR (right) of lys$E G F P^{-1+}$ mice. Of note, DN cDCs cannot be distinguished from GFP ${ }^{-} \mathrm{CD}_{11} \mathrm{~b}^{+} \mathrm{cDCs}$ due to the lack of available specific antibody. Mean percentage \pm s.d. obtained from at least three to four sections per mouse of three lys-EGFP ${ }^{-1+}$ mice. See also Supplementary Figure S4 for the location of $c D C s$ and monocyte-derived cells in Zbtb46-GFP mice. CDC, conventional dendritic cell; DN, double negative; FAE, follicle-associated epithelium; GFP, green fluorescent protein; IFR, interfollicular region; LysoDC, lysozyme-expressing dendritic cell; LysoMac, lysozyme-expressing macrophage; PP, Peyer's patch; SED, subepithelial dome. 
(Supplementary Figure S2A,B). Thus, at steady state, the only PP cDCs detectable by microscopy with CD11b were DAV cDCs. Of note, there was a good correlation between the surface expression of $\mathrm{CD} 11 \mathrm{~b}$ and its gene expression levels in the different dome phagocyte subsets (Supplementary Figure S2C). Altogether, these data indicate that $\mathrm{CD} 11 \mathrm{c}^{\mathrm{hi}}$ CD11b ${ }^{\text {hi }}$ cells of the SED are mostly constituted of LysoDCs and TIM- $4^{-}$LysoMacs, and raise the question of $\mathrm{CD} 11 \mathrm{~b}^{+} \mathrm{cDC}$ main location.

\section{$\mathrm{CD}_{11} \mathrm{~b}^{+}$dome $\mathrm{cDCs}$ are mainly located in the IFR}

We sought out to determine the location of CD11b ${ }^{+}$dome cDCs using alternative markers. We previously noticed that, although the antibody against lysozyme specifically labels LysoDCs and LysoMacs, green fluorescent protein (GFP) staining of lys-EGFP mice allows the visualization of another subset of CD11 $c^{\text {hi }}$ cells undetectable with the anti-lysozyme antibody. ${ }^{19}$ We observed that these $\mathrm{CD} 11 \mathrm{c}^{\mathrm{hi}} \mathrm{GFP}^{+}{ }^{+}$ysozyme ${ }^{-}$cells represented half of the $\mathrm{CD}_{11} \mathrm{~b}^{+}$dome cDCs (Figure 4a). In contrast, in C $x_{3} \mathrm{crl}$ $\mathrm{GFP}^{-1+}$ mice, nearly all CD11 $\mathrm{c}^{\mathrm{hi}} \mathrm{MHCII}{ }^{+} \mathrm{GFP}^{+}$cells expressed lysozyme and were LysoDCs and LysoMacs (Figure 4a). By microscopy, while most $\mathrm{GFP}^{+}$cells of the IFR of $\mathrm{Cx}_{3} \mathrm{Cr} 1-\mathrm{GFP}^{-1}$ ${ }^{+}$mice were TIM- ${ }^{+}$LysoMacs, many $\mathrm{GFP}^{+}$cells of the IFR of lys-EGFP mice did not express lysozyme nor TIM-4, indicating that they correspond to the $\mathrm{GFP}^{+} \mathrm{CD} 11 \mathrm{~b}^{+} \mathrm{cDCs}$ detected by flow cytometry (Figure $4 \mathrm{~b}$ ). As expected, these $\mathrm{GFP}^{+}$lysozyme ${ }^{-}$cells expressed SIRP $\alpha$ and CD11c (Figure 4c). Thus, at least part of $\mathrm{CD} 11 \mathrm{~b}^{+}$dome $\mathrm{cDCs}$ was located in the $\mathrm{T}$-cell zone of PPs. In addition to these $\mathrm{CD} 11 \mathrm{c}^{\mathrm{hi}} \mathrm{GFP}^{+}$ lysozyme $^{-}$cells, another important population of $\mathrm{CD} 11 \mathrm{c}^{\mathrm{hi}}$ SIRP $\alpha^{+} \mathrm{GFP}^{-}$lysozyme ${ }^{-}$cells resided in the IFR (Figure 4c). Moreover, CCR7 staining was observed in cDCs $\left(\mathrm{CD} 11 \mathrm{c}^{+}\right.$ $\mathrm{CX}_{3} \mathrm{CR} 1^{-}$cells) of the IFR but not of the SED (Figure 4d; Supplementary Figure S3). As CD $8 \alpha^{+}$dome cDCs did not express SIRP $\alpha$ (Figure 1a) and DN cDCs expressed less $\mathrm{Ccr} 7$ and more $\mathrm{Ccr} 6$ than $\mathrm{CD}_{11 \mathrm{~b}^{+}} \mathrm{cDCs}$ (Supplementary Figure S1A), CD11 $\mathrm{c}^{\text {hi }}$ SIRP $\alpha^{+} \mathrm{CCR}^{+} \mathrm{GFP}^{-}$lysozyme ${ }^{-}$cells of the IFR were likely CD11 ${ }^{+}{ }^{+} \mathrm{CDCs}$, whereas CD11 $\mathrm{c}^{\text {hi }}$ SIRP $\alpha^{+}$ $\mathrm{CCR}^{-} \mathrm{GFP}^{-}$lysozyme ${ }^{-}$cells of the SED were likely DN cDCs.
We quantified the number of cells for each subset on cryostat sections (Figure 4e). Two-third of the total SED phagocyte population was monocyte-derived cells. On the contrary, nearly three quarters (almost half SIRP $\alpha^{+}$and a quarter SIRP $\alpha^{-}$) of the total IFR phagocyte population were cDCs. The IFR contained, however, the specific population of macrophages termed TIM- $4^{+}$LysoMacs.

In agreement with these results, when Zbtb46-GFP mice were analyzed for $\mathrm{cDC}$ location, most of them $\left(\mathrm{CD} 11 \mathrm{c}^{\mathrm{hi}} \mathrm{GFP}^{+}\right.$ MerTK $^{-}$cells) were located in the IFR and serosal regions, whereas only a few were in the SED with, however, an enrichment toward crypts and base of domes (Supplementary Figure S4A-C). On the contrary, monocyte-derived cells $\left(\mathrm{CD} 11 \mathrm{c}^{\mathrm{hi}} \mathrm{GFP}^{-} \mathrm{MerTK}^{+}\right.$cells) were enriched in the SED, especially in its upper part (Supplementary Figure S4A). In general, cDCs appeared smaller and less dendritic than monocyte-derived cells. As expected, cDCs of the SED were stained for SIRP $\alpha$ but not CD11b, which was only observed on monocyte-derived cells (Supplementary Figure S4B,C). Like LysoDCs, few cDCs resided in the FAE, sometimes in close contact with LysoDCs (Supplementary Figure S4B,C). In the IFR, the major part of cDCs expressed SIRP $\alpha$ (Supplementary Figure S4C).

In conclusion, the SED, the key site of antigen uptake, is dominated by monocyte-derived cells, whereas the IFR, the major site of antigen presentation, is outnumbered by cDCs (see Figure $\mathbf{1 0}$ for a summary model).

\section{TLR7 ligand stimulation induces an increase of CD8 $\alpha^{+}$ interfollicular CDC number and migration of DAV CDCs in the IFR}

We studied the behavior of PP cDCs upon a perturbation of homeostasis through detection of an activation signal in vivo. R848 is a TLR7 agonist known to drastically alter intestinal DC distribution, migration, and activation, including those of PPs. ${ }^{12}$ Alteration induced by R848 did not strongly interfere with our PP phagocyte gating strategy (Supplementary Figure S5A). We observed that the absolute number of $\mathrm{CD} 8 \alpha^{+} \mathrm{cDCs}$ doubled $9 \mathrm{~h}$ after R848 gavage of C57BL/6 mice, whereas those

Figure 5 Migration of CD11b ${ }^{+}$DAV cDCs in the IFR of R848-fed mice. (a) Fold change of absolute number of cDCs extracted from PP collected 0, 4, 9, and $16 \mathrm{~h}$ after gavage of C57BL/6 mice with R848. Number of CD $8 \alpha^{+}$CDCs doubled between 4 and $9 \mathrm{~h}$ after adjuvant treatment. ${ }^{*} P<0.05$; unpaired $T$-test with Welch's correction performed from data of three $(4 \mathrm{~h})$ to six independent experiments $(9 \mathrm{~h})$. (b) Left: expression of CD8 $\alpha$ and CD11b in $\mathrm{CD}_{11 \mathrm{C}^{\mathrm{hi}} \mathrm{MHCII}}{ }^{\mathrm{BST}} 2^{-} \mathrm{CDCs}$ extracted from C57BI/ 6 mice PP collected 0 and $9 \mathrm{~h}$ after gavage with R848. R848 induced an increase of CD8 $\alpha^{+} \mathrm{cDC}$ percentage among CDCs. Right: ratio of the different dome CDCs before and after $9 \mathrm{~h}$ of R848 treatment (mean percentage \pm s.d. of five independent experiments). (c) Confocal microscopy projection of an IFR from a C $x_{3} C r 1-G F P^{-1+}$ mouse PP taken $9 \mathrm{~h}$ after R848 gavage and stained for GFP (green), CD11c (red), and CD11b (magenta). R848 induced the recruitment of CD11c ${ }^{+} \mathrm{CX}_{3} \mathrm{CR}^{-}{ }^{-} \mathrm{CD}_{11} \mathrm{~b}^{\mathrm{hi}}$ cells in the IFR. Bar, $20 \mu \mathrm{m}$. (d) Normalized mean relative expression \pm s.d. of $C d 101$ in PP and villus (data from Immgen database) phagocyte populations. Unlike CD11b ${ }^{+}$villus $\mathrm{cDCs}, \mathrm{CD}_{11 \mathrm{~b}}{ }^{+}$dome cDCs did not express Cd101. (e) Expression of CD101 in dome and DAV of PP. Upper row: CD101 was expressed by DAV CD11b ${ }^{+}$CDCs but not by

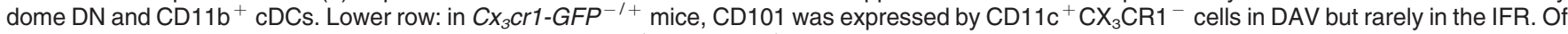
note, CD101 was also expressed by some DAV T cells (CD3e ${ }^{+} \mathrm{CD} 11 \mathrm{c}^{-}$or + cells; see Supplementary Figure S6). (f) Confocal microscopy projection of an IFR from a $\mathrm{Cx}_{3} \mathrm{Cr}_{1-G F P}{ }^{-1+}$ mouse PP taken $9 \mathrm{~h}$ after R848 gavage and stained for GFP (green), CD11C (red), and CD101 (magenta). R848 induced the recruitment of $\mathrm{CD} 11 \mathrm{c}^{+} \mathrm{CX}_{3} \mathrm{CR} 1^{-} \mathrm{CD} 101^{+}$cells in the IFR. Bar, $20 \mu \mathrm{m}$. See also Supplementary Figure $\mathbf{S 6}$. (g,h) Confocal microscopy projection of PP sections from $\mathrm{Cx}_{3} \mathrm{Cr} 1-\mathrm{GFP}{ }^{-1+}$ mice taken $5 \mathrm{~h}$ after R848 gavage and stained for GFP (green), CD11c (red), CD4 (cyan), and (g) CD101 or (h) CCR7 (magenta). R848 induced the appearance of $\mathrm{CD} 11 \mathrm{c}^{+} \mathrm{CD} 101^{+}$and $\mathrm{CD} 11 \mathrm{c}^{+} \mathrm{CCR} 7^{+}$cells (arrowheads) at the base and in the crypts of DAV in the vicinity of the IFR. Bar, $20 \mu \mathrm{m}$. See also Supplementary Figures S3 and S6. C, crypt; cDC, conventional dendritic cell; DAV, dome-associated villus; DN, double negative; F, follicle; FAE, follicle-associated epithelium; IFR, interfollicular region; PP, Peyer's patch; SED, subepithelial dome. 
of $\mathrm{DN}$ and $\mathrm{CD} 11 \mathrm{~b}^{+}$dome $\mathrm{cDCs}$ remained stable (Figure 5a). Accordingly, the proportion of CD $8 \alpha^{+}$cDCs among PP cDCs increased from $16.5 \pm 2.1$ to $25.1 \pm 5.3 \%$ (Figure $5 \mathbf{b}$ ).

Surprisingly, although villus cDCs are known to massively and rapidly migrate to the mesenteric lymph nodes (MLNs) upon R848 treatment, ${ }^{12}$ we did not observe any loss of DAV cDCs after tissue dissociation (Figure $\mathbf{5 a}, \mathbf{b}$ ). In parallel, we observed a strong recruitment of $\mathrm{CD} 11 \mathrm{c}^{\mathrm{hi}} \mathrm{CX}_{3} \mathrm{CR} 1^{-} \mathrm{CD} 11 \mathrm{~b}^{\text {hi }} \mathrm{cDCs}$ in the IFR (Figure $5 \mathbf{c}$ to be compared with Figure $\mathbf{3 b}$ ). In order to determine whether these newly recruited interfollicular cDCs were coming from DAV, we investigated the expression of CD101, which gene was expressed by CD11 ${ }^{+} \mathrm{cDCs}$ of the villus
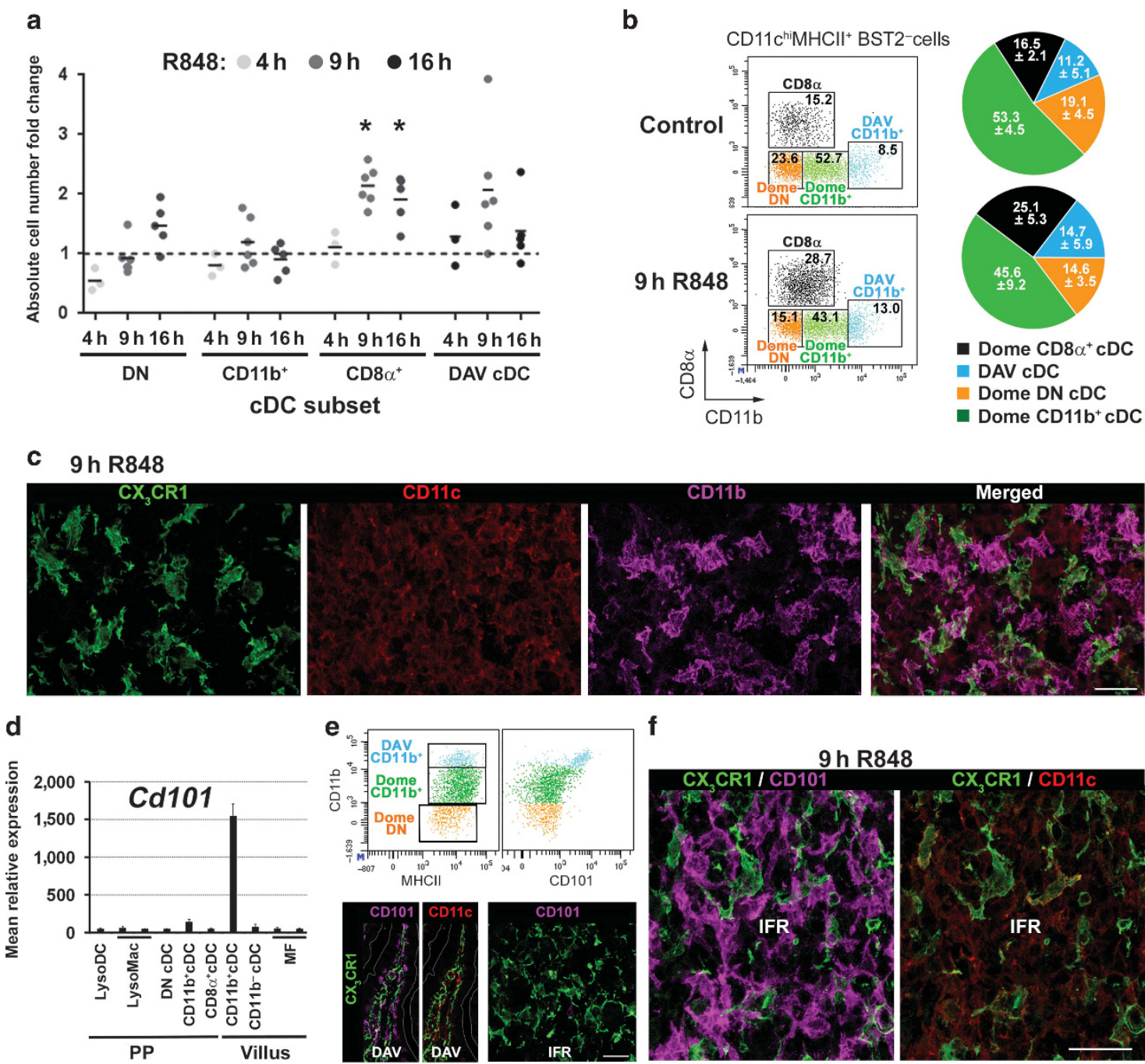

9

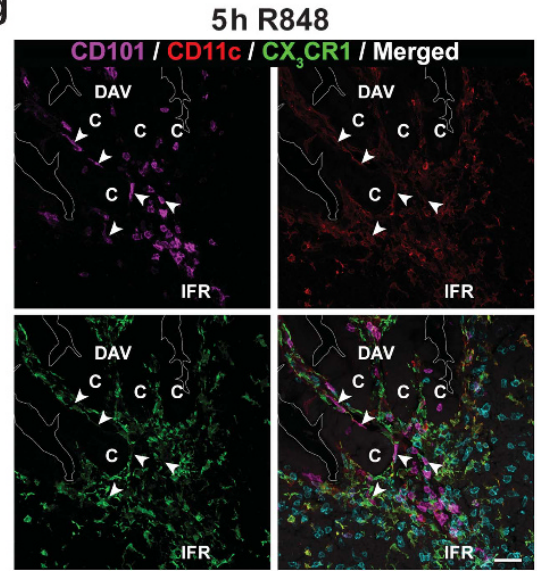

h

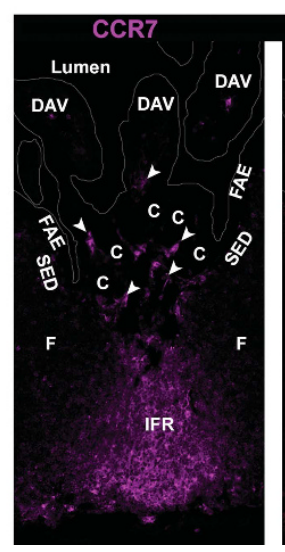

5h R848

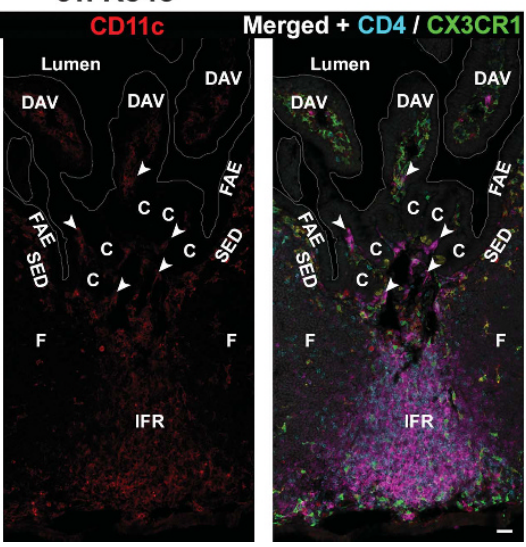

Figure 5 See for caption page on 1419. 
but not of the dome (Figure 5d). At steady state, CD101 was expressed by DAV but not by interfollicular cDCs (Figure 5e; Supplementary Figure S6). However, $\mathrm{CD}_{11 \mathrm{c}^{+} \mathrm{CX} 3 \mathrm{CR} 1^{-}}$ $\mathrm{CD} 01^{+} \mathrm{cDCs}$ were massively recruited in the IFR upon R848 treatment, whereas there were very few left in DAV as compared to untreated PP (Figure 5f; Supplementary Figure S6). There was an anatomic continuity between the base of DAV and the IFR, and we observed the appearance of CD11c ${ }^{+}$CX3CR $1{ }^{-} \mathrm{CD} 101^{+} \mathrm{cDCs}$ at the base of DAV and in DAV crypts from $5 \mathrm{~h}$ of R848 treatment onwards (Figure 5g). This indicates that the recruitment of cDCs from DAV to the IFR occurred through migration across the base and the crypts of DAV. Interestingly, CCR7 expression was upregulated in DAV cDCs (Supplementary Figure S5B) and appeared in DAV crypt-located $\mathrm{CD} 11 \mathrm{c}^{+} \mathrm{CX}_{3} \mathrm{CR} 1^{-}$cDCs (Figure 5h; Supplementary Figure S3). Altogether, these data indicate that, following R848 treatment, the IFR is a site of DAV cDC migration.

\section{TLR7 ligand induces the activation of both $\mathrm{CD} 11 \mathrm{~b}^{+}$and CD8 $\alpha^{+}$interfollicular cDCs.}

R848 treatment induced an alteration of $\mathrm{CD} 8 \alpha^{+} \mathrm{cDC}$ phenotype with an increase of MHCII and a slight decrease of CD11c surface expression (Figure 6a). Although less pronounced, a similar activation profile was observed for $\mathrm{CD}_{11 \mathrm{~b}}{ }^{+}$dome cDCs.

At steady state, PP CD $8 \alpha^{+}$cDCs can be detected using CD205. ${ }^{14}$ We confirmed that CD205 was expressed by all $\mathrm{CD} 8 \alpha^{+}$cDCs but not or weakly by SIRP $\alpha^{+}$cDCs (Figure 6b). However, upon R848 gavage, one-third of $\mathrm{CD}_{11 \mathrm{~b}^{+}}$dome cDCs but very few DN dome cDCs acquired CD205. Moreover, expression of $\mathrm{CD} 205$ on $\mathrm{CD} 11 \mathrm{~b}^{+}$dome cDCs was correlated with their state of activation (Figure $\mathbf{6 b}$ ). This confirmed the immature status of $\mathrm{DN}$ as compared to $\mathrm{CD}_{11 \mathrm{~b}}{ }^{+} \mathrm{cDCs}$. By confocal microscopy, CD205 was absent from the SED of R848treated mice, indicating that R848 did not induce the recruitment of $\mathrm{CD} 8 \alpha^{+}$and activated $\mathrm{CD} 205^{+} \mathrm{CD} 11 \mathrm{~b}^{+}$ cDCs in the SED (Figure 6c). However, CD205 was strongly expressed in the IFR of R848-fed mice, indicating that both activated $\mathrm{CD} 8 \alpha^{+} \mathrm{cDCs}$ and $\mathrm{CD} 11 \mathrm{~b}^{+} \mathrm{cDCs}$ reside in the IFR (Figure 6d). The activated status of interfollicular cDCs was further confirmed by the strong increase of the staining for CCR7, the activation marker CD83, and the co-stimulatory molecule CD86 in the IFR of R848-treated mice (Supplementary Figures S3 and S7A,B).

R848-induced PP CDC stimulation is indirect and mediated at least in part through a TNF-dependent mechanism

TLR7 messenger RNA and protein were detected in PP monocyte-derived cells (LysoDCs and LysoMacs) and pDCs but not in cDCs (Figure 7a,b). Migration and activation of PP cDCs is thus likely to be indirect. We previously showed that LysoDCs and LysoMacs produce and secrete TNF upon R848 stimulation in vitro. ${ }^{17}$ Moreover, Yrlid et al. ${ }^{12}$ have shown that R848-induced migration of villus DCs to the MLN is dependent on TNF. We thus investigated the role of TNF in PP CDC migration and activation processes. As previously reported, migration of villus DCs to the MLN was impaired when TNF signaling was neutralized (Figure 7c). In PP, TNF inhibition prevented the increase of $\mathrm{CD} 8 \alpha^{+} \mathrm{cDC}$ number and strongly decreased interfollicular $\mathrm{cDC}$ activation (Figure $\mathbf{7 d , e )}$. In addition, the recruitment of CD101 ${ }^{+} \mathrm{DAV} \mathrm{cDC}$ in the IFR was blocked by anti-TNF treatment (Figure 7e). Therefore, both migration and to some extent activation of dome and DAV cDCs were dependent on TNF.

\section{The genetic reprograming of R848-activated interfollicular cDCs converges toward steady-state PP monocyte-derived cell transcriptional profiles and induces the expression of both immuno-stimulatory and -inhibitory molecules}

As activated cDCs display a distinct phenotypical pattern (Figure 6a), quintuplicates of activated $\mathrm{CD} 8 \alpha^{+} \mathrm{cDCs}$ and triplicates of activated $\mathrm{CD}_{11} \mathrm{~b}^{+} \mathrm{cDCs}$ were isolated and submitted to mouse whole-genome microarray analysis. Generated data were combined with those of phagocytes from unstimulated mice. $\mathrm{CD} 8 \alpha^{+}$cDCs were much more impacted by R848 treatment than CD11b ${ }^{+}$cDCs with 1,052 DEGs as compared to 356 DEGs for CD11b ${ }^{+}$cDCs (Figure 8a). Fifty-three DEGs between activated and resting cDCs belonged to the core gene signature associated with activated DCs as defined by Vu Manh et al. ${ }^{5}$ (Supplementary Table S3). A striking feature was the induction and repression in both cDC subsets of interferon-stimulated and -inhibited genes,

Figure 6 Phenotype and location of activated dome cDCs in R848-fed mice. (a) Alteration of CD11c and MHCII expression in CD11b ${ }^{+}$and CD8 $\alpha^{+}$

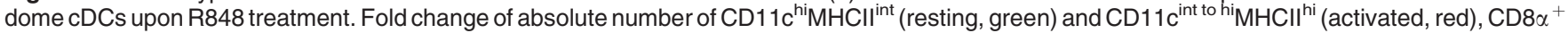
(upper panel), and CD11 b ${ }^{+}$(lower panel) dome cDCs extracted from PP collected $0,4,9$, and $16 \mathrm{~h}$ after gavage of C57BL/6 mice with R848 and gated as shown on the left dot plots. Number of cDCs with an activated profile strongly increased upon R848 treatment. ${ }^{*} P<0.05$; ${ }^{*} P<0.01$; unpaired $T$-test with Welch's correction performed from data of three $(4 \mathrm{~h})$ to five independent experiments ( 9 and $16 \mathrm{~h}$ ). (b) Expression of CD205 in DN (orange) and CD8 $\alpha^{+}$ dome cDCs (black) and total (dark green, upper row) or resting (CD11 $\mathrm{c}^{\text {hi }} \mathrm{MHCI}{ }^{\text {int }}$, light green, lower row) and activated (CD11 $\mathrm{c}^{\text {int to hi }} \mathrm{MHCI} \mathrm{I}^{\text {hi }}$, red, lower row) $\mathrm{CD} 11 \mathrm{~b}^{+}$dome $\mathrm{CDC}$ s at steady state and 9 or $16 \mathrm{~h}$ after R848 treatment. Resting and activated CD $11 \mathrm{~b}^{+} \mathrm{cDC}$ were gated as shown in $\mathbf{a}$. The lower left histogram shows the percentage \pm s.d. of CD205 ${ }^{+}$cells among DN (orange), resting (light green), and activated (red) CD11b ${ }^{+}$cells at steady state and 9 or $16 \mathrm{~h}$ after R848 treatment. CD205 was constitutively expressed by CD8 $\alpha^{+} \mathrm{cDCs}$, absent from DN cDCs and induced by R848 on CD11b ${ }^{+} \mathrm{cDCs}$. ${ }^{*} P<0.05$; ${ }^{* *} P<0.01$; unpaired $T$-test with Welch's correction performed from data of three independent experiments. (c,d) Confocal microscopy projection of PP sections from $\mathrm{Cx}_{3} \mathrm{Cr}_{1-G F P^{-1+}}$ mice taken $9 \mathrm{~h}$ after R848 gavage and stained for GFP (green), CD11c (red), lysozyme (yellow), CD4 (cyan), CD45R (orange), and CD205 (magenta). In R848-treated mice, CD205 was expressed by interfollicular (d) but not by subepithelial $\mathrm{CD} 11 \mathrm{c}^{+} \mathrm{CX}_{3} \mathrm{CR} 1^{-}$cells (c). The same magnification is shown in both images. Bar, $20 \mu \mathrm{m}$. See also Supplementary Figure $\mathbf{S 7}$ for other activated DC marker staining. Act, activated; $C D C$, conventional dendritic cell; DN, double negative; F, follicle; FAE, follicle-associated epithelium; IFR, interfollicular region; Rest, resting; SED, subepithelial dome. 
respectively. (Figure 8a). A set of chemokine genes was induced in stimulated cDCs (Ccl19 for CD8 $\alpha^{+}$cDCs; Ccl8, Cxcl9, and Cxcl10 for $\mathrm{CD} 11 \mathrm{~b}^{+}$cDCs; and $\mathrm{Ccl} 5$ and $\mathrm{Ccl} 22$ for both subsets; Figure 8a,b). CCL22 was indeed strongly expressed in the IFR of R848-fed mice (Supplementary Figure S7C). Il15 was the main cytokine gene to be upregulated in both subsets upon stimulation (Figure 8a). Interestingly, genes involved in cell migration were drastically downregulated in both activated
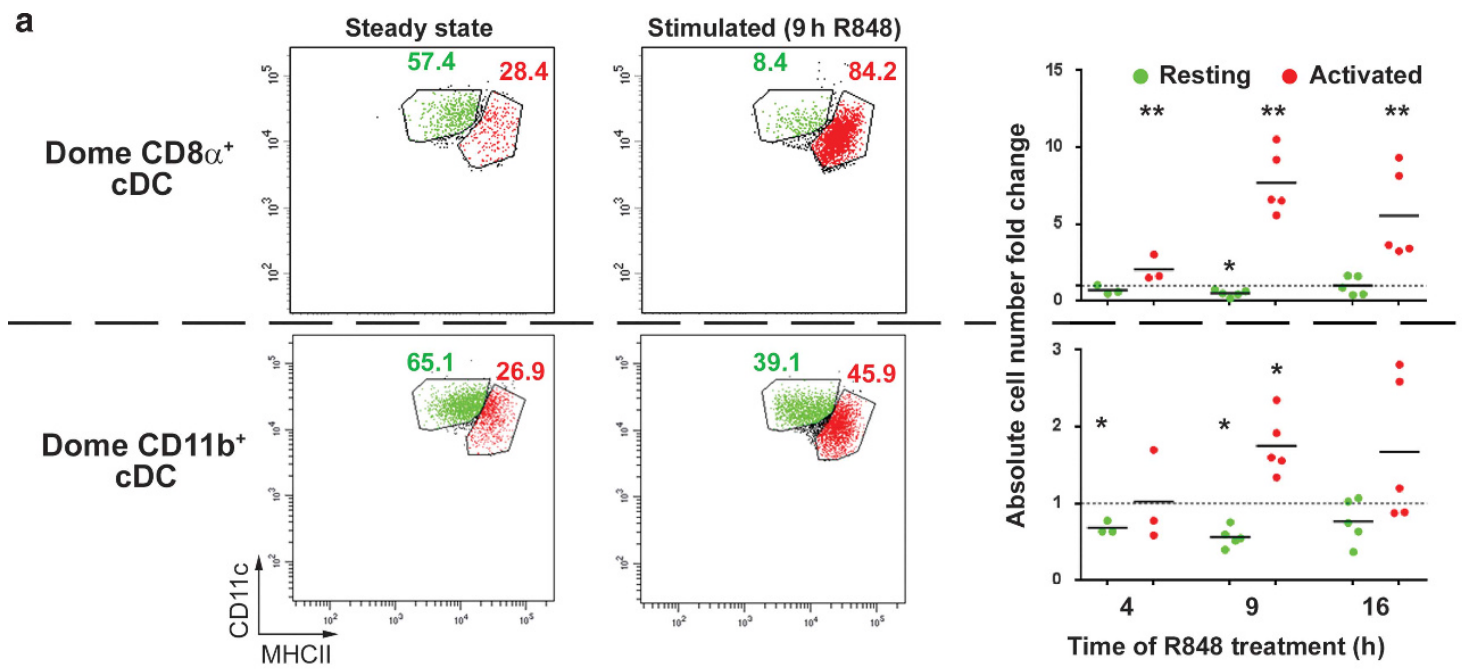

b
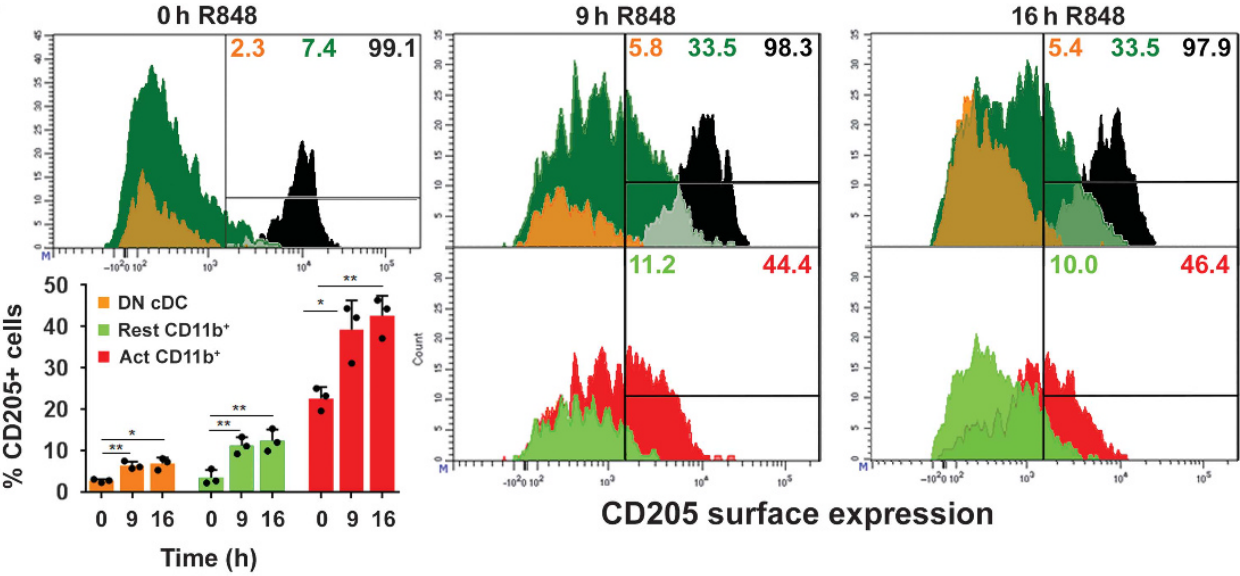

- $\mathrm{CD}_{11} \mathrm{~b}^{+} \mathrm{cDC}$

- DN cDC

- $\mathrm{CD} 8 \alpha^{+} \mathrm{cDC}$
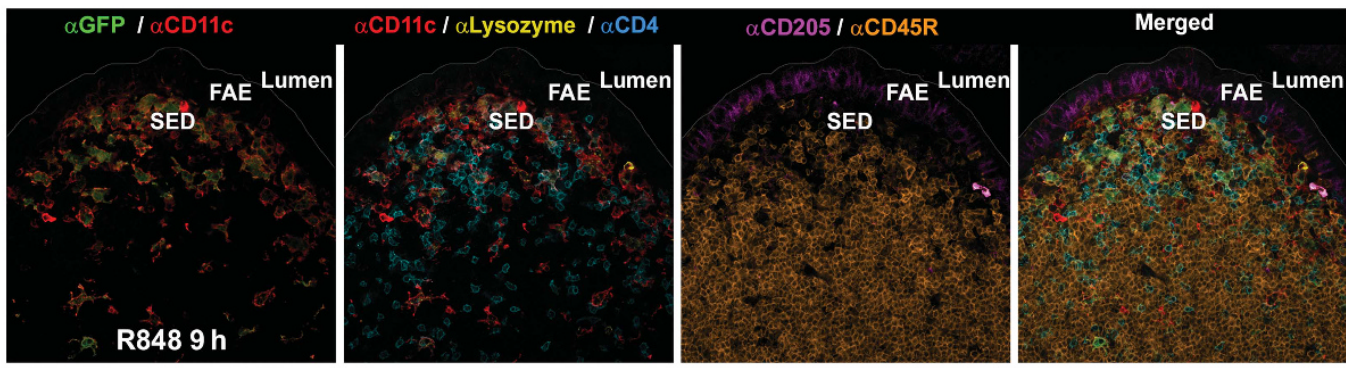

d
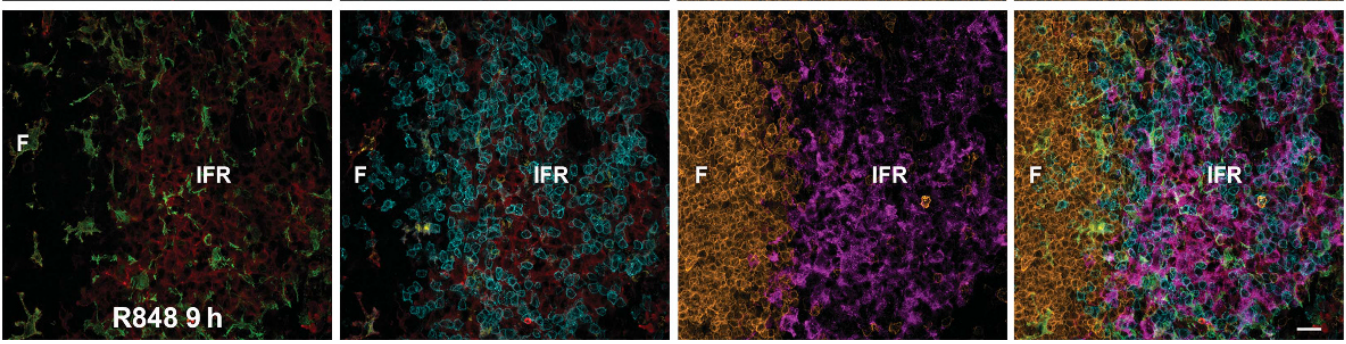

Figure 6 See for caption page on 1421. 
a

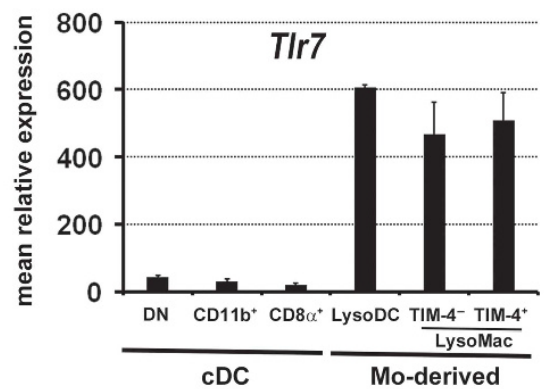

b
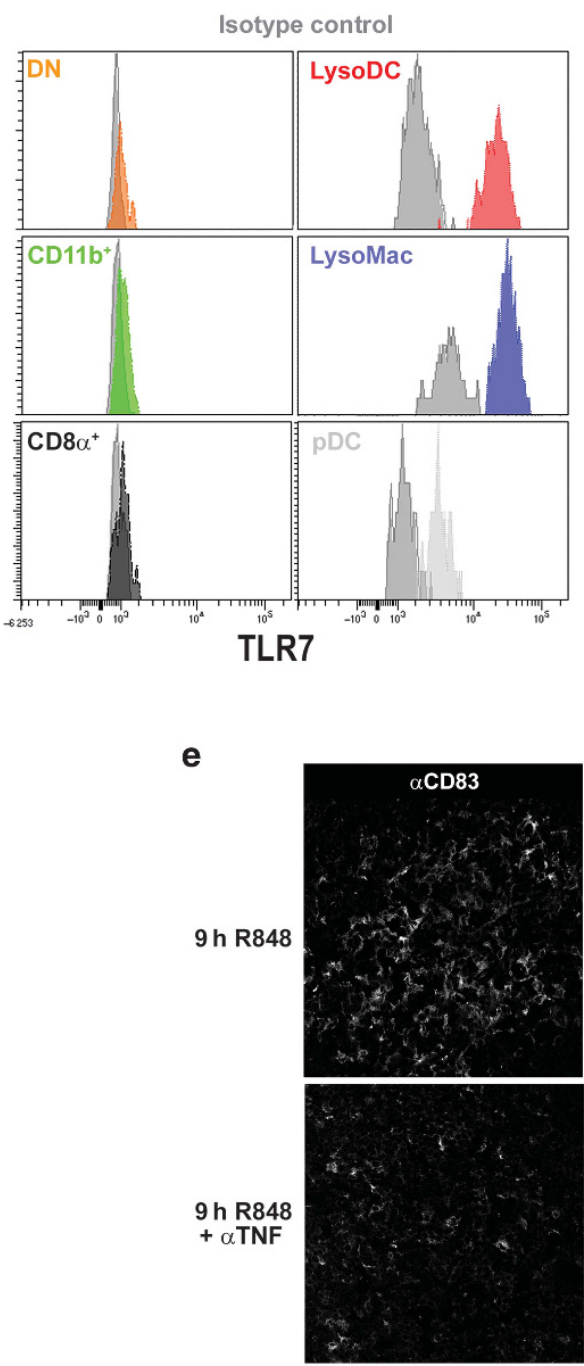

C

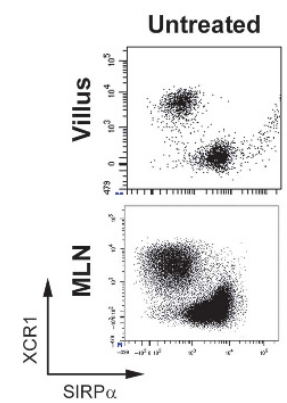

d
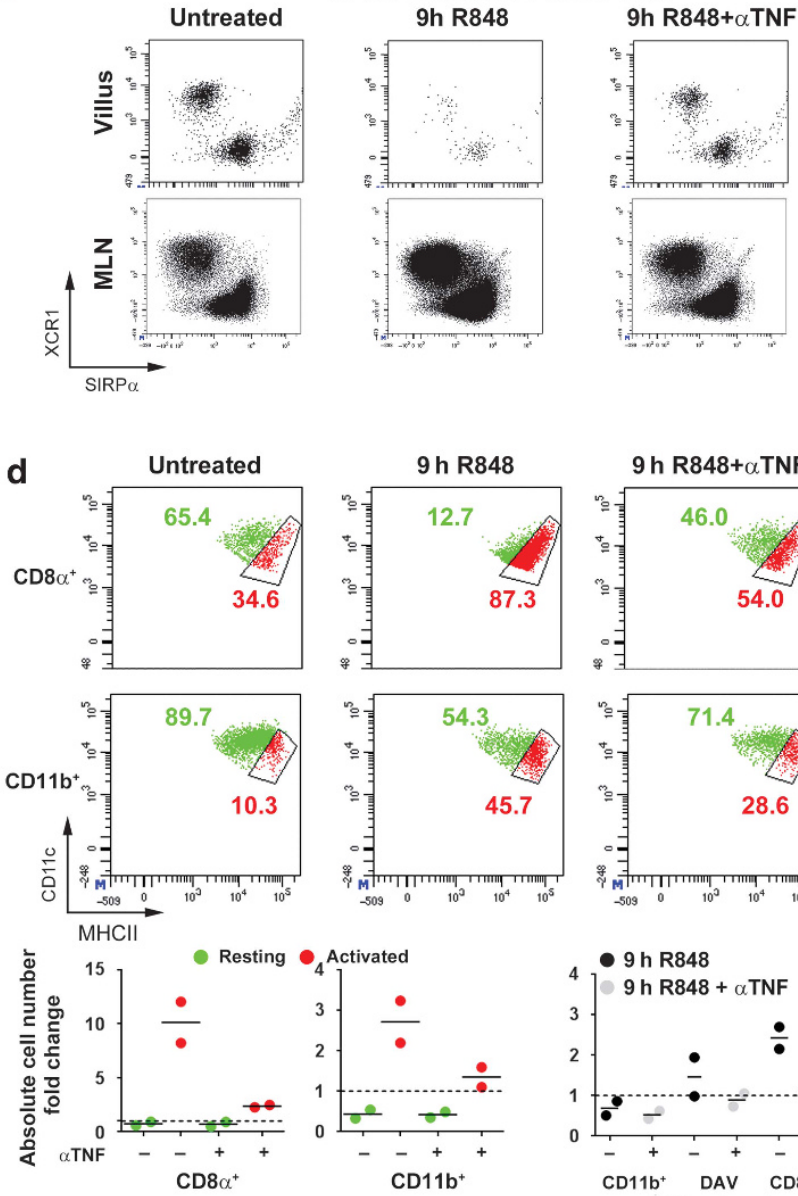

$9 \mathrm{~h} \mathrm{R848+ \alpha TNF}$
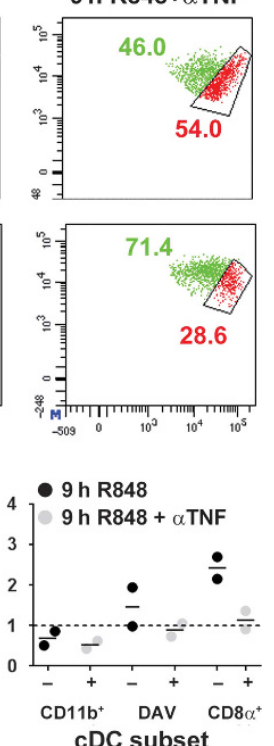

cDC subset
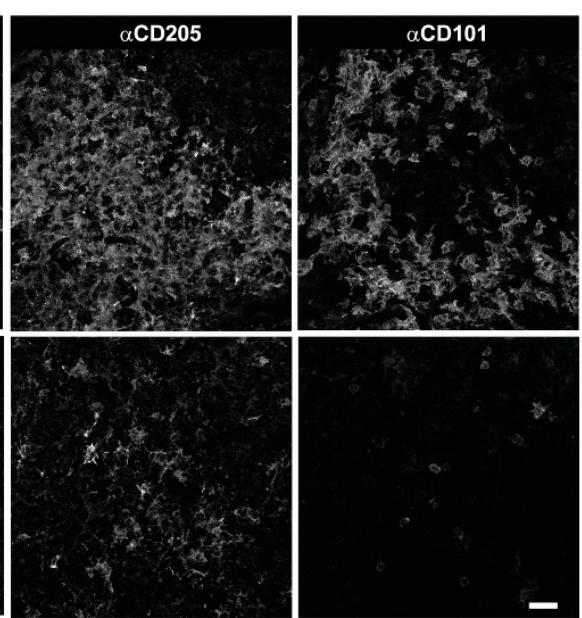

Figure 7 TNF dependency of R848-induced migration, number increase, and activation of DAV and dome cDCs. (a) Normalized mean relative expression \pm s.d. of TIr7 in PP phagocytes. TIr7 was expressed by monocyte-derived cells but not by CDCs. (b) Intracellular expression of TLR7 in PP phagocytes. TLR7 was expressed by LysoDCs, LysoMacs, and pDCs but not by cDCs. (c-e) Effect of TNF inhibition on migration, increased number, and activation of cDCs from villus, MLN, and PP. Data are representative of two independent experiments.(c) R848-induced migration of villus cDCs to the MLN was impaired upon anti-TNF antibody treatment. Representative dot plot of CDC total population in the villus (upper row) and in the MLN (lower row) of untreated (left), R848-treated (middle), and R848 + anti-TNF-treated mice (right). (d) R848-induced activation of CD8 $\alpha^{+}$(first row) and CD11b ${ }^{+}$ (second row) dome cDCs was impaired in anti-TNF-treated mice. Lower left: absolute cell number fold change of resting (green) and activated (red) $\mathrm{CD} 8 \alpha^{+}$(left), and CD11b ${ }^{+}$(right) dome cDCs in R848-treated mice. Lower right: absolute cell number fold change of CD11 b ${ }^{+}$dome and DAV cDCs, and $\mathrm{CD} 8 \alpha^{+}$dome cDCs. Two independent experiments are shown. (e) Confocal microscopy projection of PP from C57BI/6 mice treated (bottom) or not (top) with a TNF-blocking antibody and fed with R848 for $9 \mathrm{~h}$. Cryostat sections were stained for CD83, CD205, and CD101. Anti-TNF treatment causes a loss of the R848-induced activation (CD83 and CD205) and recruitment (CD101) of interfollicular and DAV CDCs, respectively. The same magnification is shown in all images. Bar, $20 \mu \mathrm{m}$. cDC, conventional dendritic cell; DAV, dome-associated villus; LysoDC, lysozyme-expressing dendritic cell; LysoMac, lysozyme-expressing macrophage; MLN, mesenteric lymph nodes; PP, Peyer's patch; TNF, tumor necrosis factor. 
a

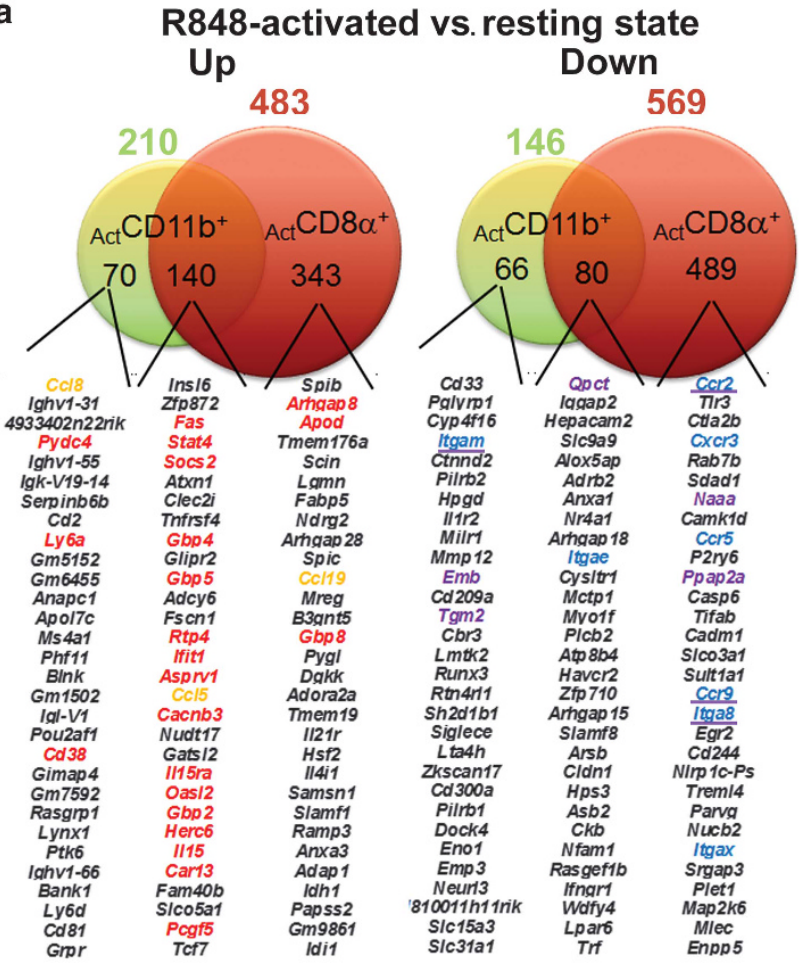

ISGs Chemoattraction IIGs Cell migration / adhesion b

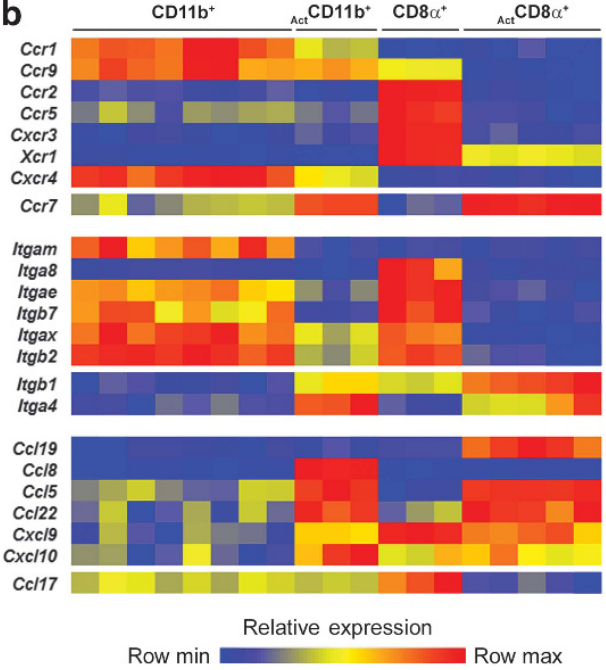

d

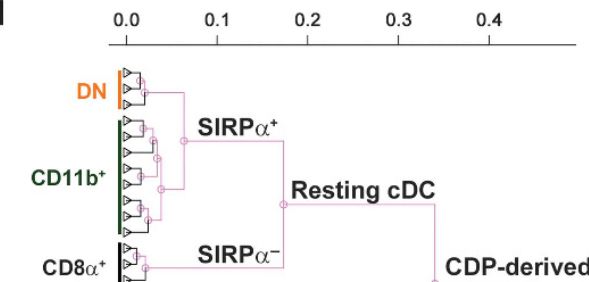

C

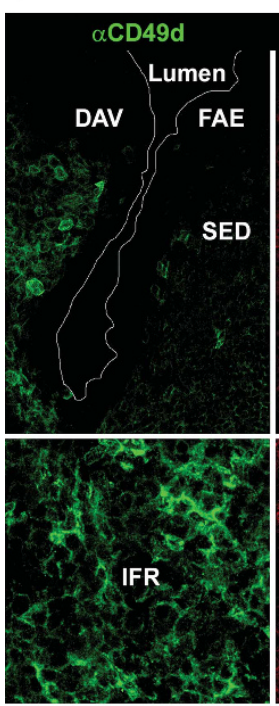

9 h R848

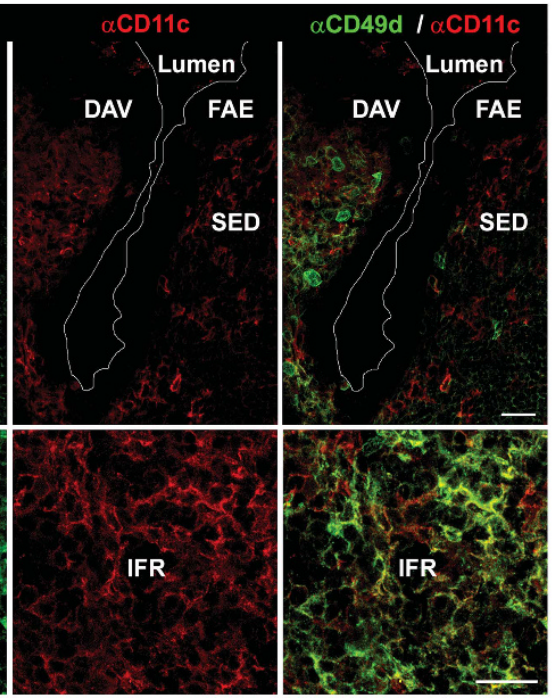

${ }_{\text {Act }} \mathrm{CD} 8 \alpha^{+}$

Activated cDC

${ }_{\text {Act }} \mathrm{CD} 11 \mathrm{~b}^{+}$

LysoDC

LysoMac Monocyte-derived

e

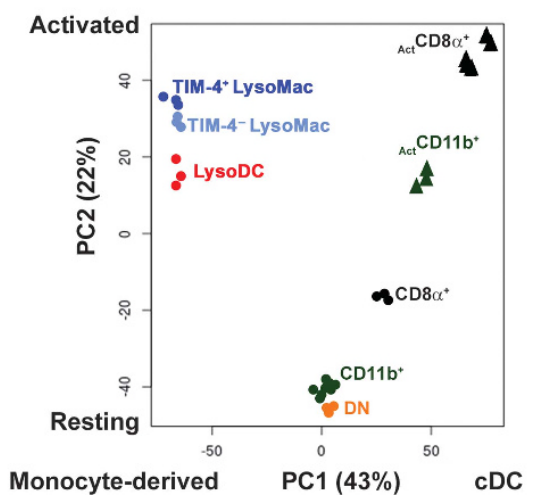

Figure 8 Genetic reprograming of PP cDCs upon R848 stimulation. (a) Venn diagrams and most up- and downregulated genes between R848activated and resting $\mathrm{CD} 11 \mathrm{~b}^{+}$and $\mathrm{CD} 8 \alpha^{+} \mathrm{CDC}$ subsets are shown. Integrin and chemokine receptor genes are in blue, chemokine genes in orange, and ISGs and IIGs in red and purple, respectively. See also Supplementary Table S3. (b) Heatmap of chemokine receptor, integrin, and chemokine gene expression in resting vs. activated CD11 $\mathrm{b}^{+}$and CD8 $\alpha^{+} \mathrm{cDC}$ subsets is shown. (c) Confocal microscopy projection of a PP from a C57BL/6 mouse $9 \mathrm{~h}$ after R848 gavage. Cryostat sections were stained for CD49d (green) and CD11c (red). Top: CD49d (encoded by Itga4) was strongly expressed by DAV but not SED cells. Bottom: unlike CD11c ${ }^{+}$cells of the SED, most CD11c ${ }^{+}$cells of the IFR expressed high levels of CD49d. Bars, $20 \mu \mathrm{m}$. (d) Hierarchical clustering of PP phagocytes, by Pearson correlation distance and Ward's aggregation, after selection of genes with a differential expression between any subset $>1.5$ (8,558 probes). (e) Principal component analysis of resting and activated PP phagocytes is shown. See also Supplementary Table S4. Data are from three to five independent cell-sorting experiments with pooled cells from 42 mice per experiment combined with our previous gene array data deposited into Gene Expression Omnibus repository under accession number GSE65514. See also Supplementary Figure S8. Act, activated; CDC, conventional dendritic cell; DAV, dome-associated villus; FAE, follicle-associated epithelium; IFR, interfollicular region; IIG, interferon-inhibited gene; ISG, interferon-stimulated gene; PP, Peyer's patch; SED, subepithelial dome. 

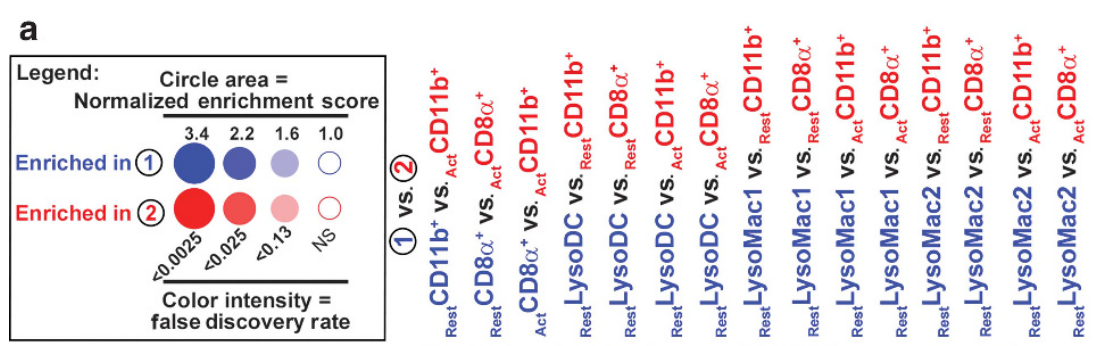

b

$\begin{array}{lllllllllll} & \\ 0\end{array}$

Type I ISGs
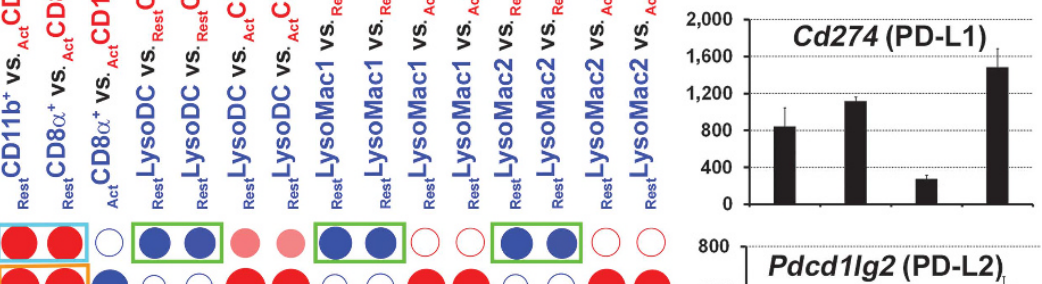

Migration

10

Migration

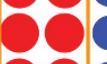

Migration

Activation 0

Immune response $\left\{\begin{array}{lll}+ & + & 0 \\ - & 0 & 0\end{array}\right.$
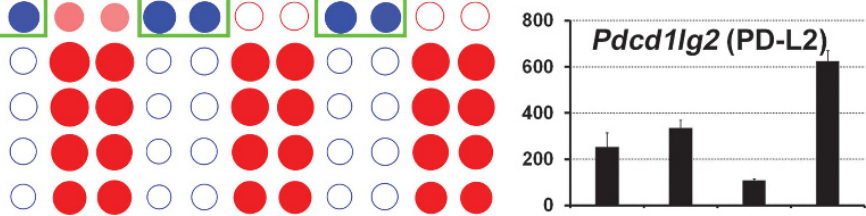

${ }^{R 848} \frac{0 \quad 9 h}{\mathrm{CD}^{2} 1 \mathrm{~b}^{+}} \frac{0 \quad 9 \mathrm{~h}}{\mathrm{CD}^{+} \mathrm{a}^{+}}$
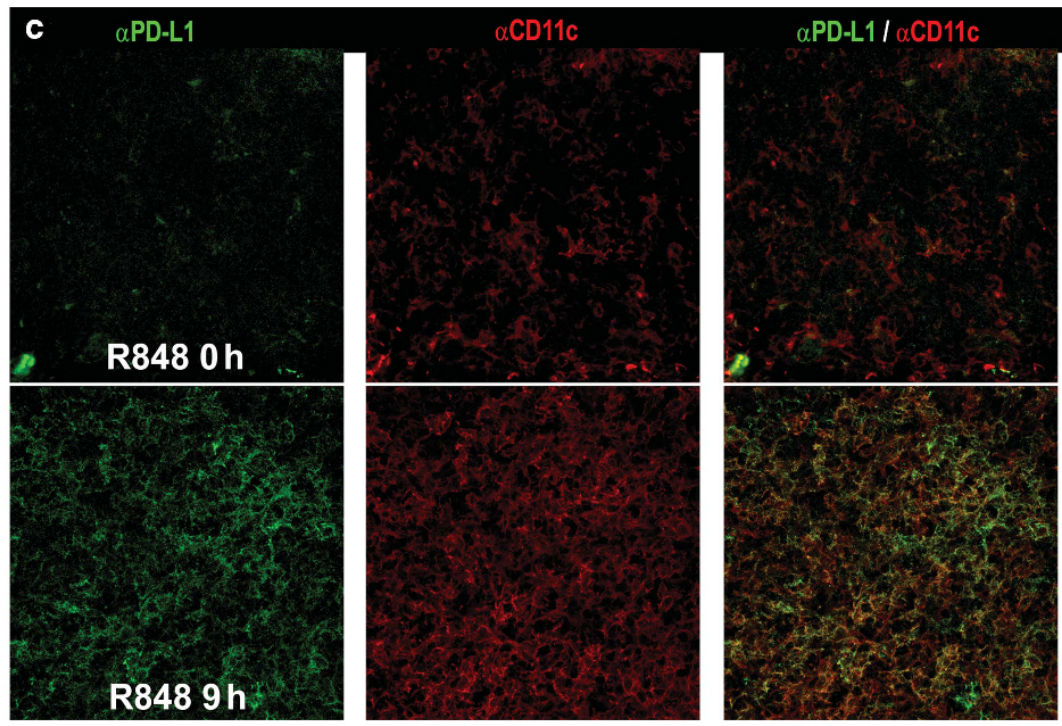

$\alpha$ PD-L1 / aCD11c / aCD8 / aCD4
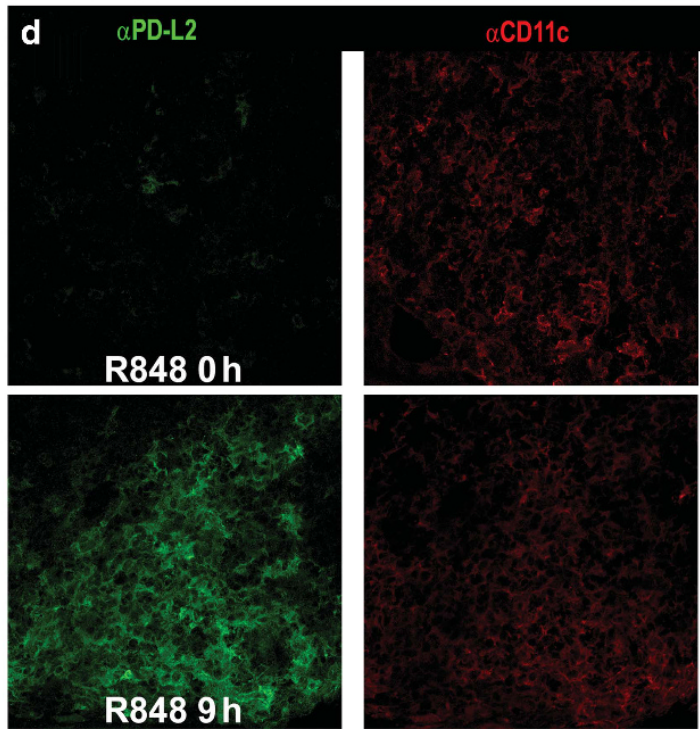

aPD-L2 / aCD11c
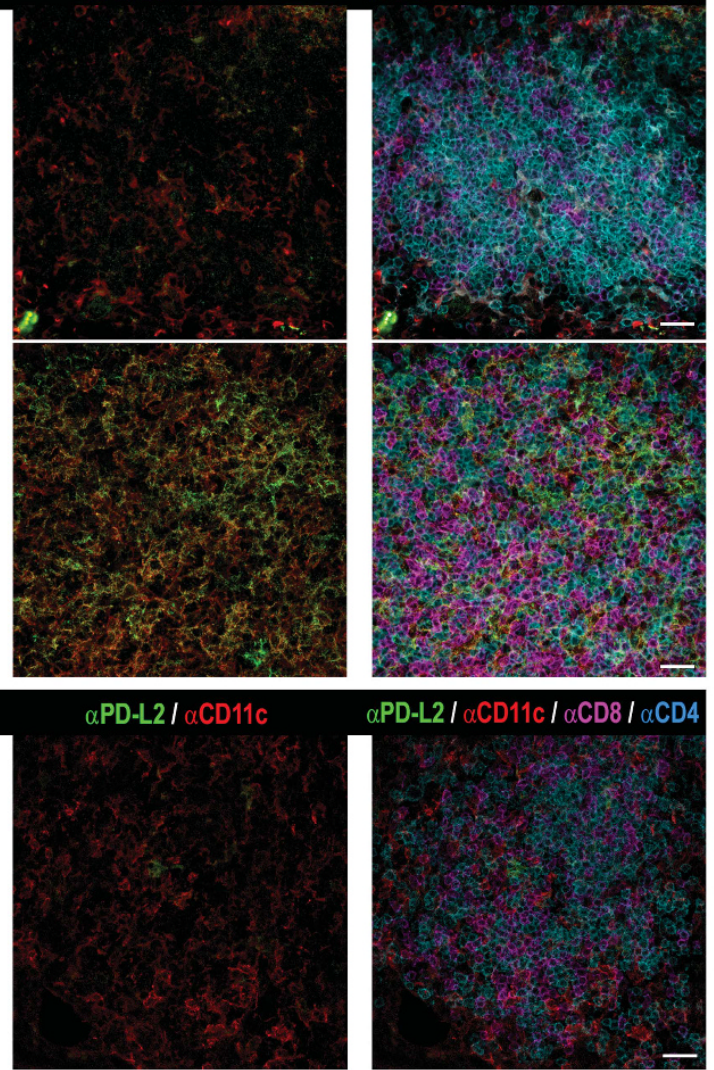

aPD-L2 / aCD11c / aCD8 / aCD4

Figure 9 See for caption page on 1426.
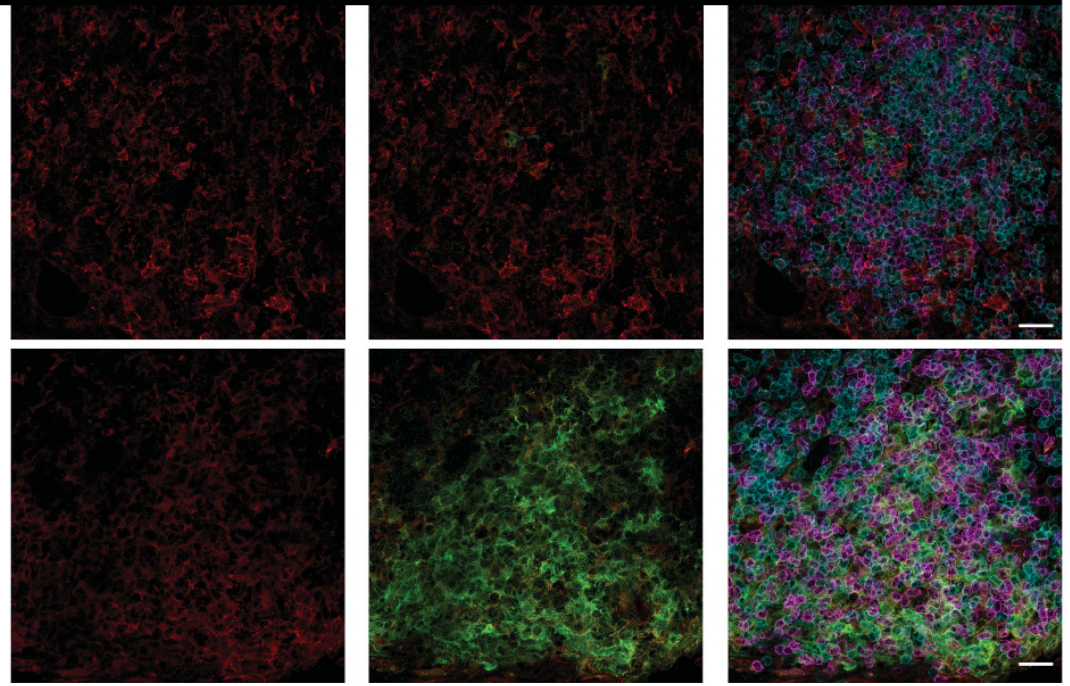
cDC subsets at the notable exception of $\mathrm{Ccr} 7$ and of the two integrin genes Itga4 and Itgb1, which proteins (CD49d and CD29) form the integrin complex VLA4 (Figure 8a,b). In agreement with these data, CCR7 and CD49d were strongly expressed in interfollicular cDCs (Figures $5 \mathbf{h}$ and $\mathbf{8 c}$; Supplementary Figure S3).

Activation tended to attenuate CDC subset identity as both R848-activated CD11b ${ }^{+}$and CD8 $\alpha^{+}$cDCs clustered together apart from their resting counterparts (Figure 8d). Accordingly, genes encoding key general cDC markers (e.g., CD11c, CD103, and c-Kit) or markers of $\mathrm{CD}_{11 \mathrm{~b}}{ }^{+}$(e.g., CD11b, CLEC4A4, and M-CSFR) and CD8 $\alpha^{+}$(e.g., XCR1, CLEC9A, and TLR3) cDCs were strongly downregulated in activated as compared to resting state (Figure 8a; Supplementary Figure S8A). Although still detectable, we indeed observed a decrease of XCR1 molecules at the surface of activated $\mathrm{CD} 8 \alpha^{+}$cDCs (Supplementary Figure S8B). As the expression of Sirpa and Cd8a was not or weakly altered (Supplementary Figure S8A), they represent key markers to distinguish PP SIRP $\alpha^{-}$ $\left(\mathrm{CD} 8 \alpha^{+}\right)$from SIRP $\alpha^{+}\left(\mathrm{DN} / \mathrm{CD} 11 \mathrm{~b}^{+}\right) \mathrm{cDCs}$ whatever their state of activation. Upon activation, CD101 was not induced in $\mathrm{CD}_{11 b^{+}}$dome cDCs, thus confirming the DAV origin of $\mathrm{CD} 101^{+}$interfollicular cDCs in R848-fed mice (Supplementary Figure S8C).

In principal component analysis, the second principal component separated resting from activated cDCs (Figure 8e). Interestingly, steady-state PP monocyte-derived cells (i.e., LysoDCs and LysoMacs) segregated with activated $\mathrm{cDCs}$ on this axis, suggesting that the former displayed a constitutive activated state. Accordingly, there was a main contribution to this axis of interferon-stimulated genes (e.g., GBP family of genes, Irf7, Rsad2, Zbp1, Oasl1, Oasl2, and Vcam 1; Supplementary Table S4) expressed by steady-state PP monocyte-derived cells. ${ }^{17}$ Sixty-three upregulated genes in resting PP monocyte-derived cells as compared to resting cDCs became upregulated in activated cDCs as compared to resting cDCs (Supplementary Table S3), confirming this shift of activated cDCs toward a genetic program already imprinted in steady-state PP monocyte-derived cells.

BubbleGUM analysis (Figure 9a; Supplementary Table S2) confirmed the enrichment of type I interferon-stimulated genes in resting LysoDCs and LysoMacs (green boxes) and in activated PP cDCs (blue box) as compared to resting cDCs. As expected, $\mathrm{CDC}$ migration and activation gene signatures were enriched in activated as compared to resting PP cDCs (orange box, Figure 9a). Surprisingly, in addition to genes linked to the activation of the immune response, those related to its control were also enriched in activated PP cDCs as compared to their resting counterparts (Figure 9a, black box). In order to confirm this early upregulation of immunomodulatory molecules in R848-treated mice, we investigated the expression of two major T-cell activation suppressors, PD-L1 and PD-L2, which transcripts were strongly upregulated in $\mathrm{CD} 8 \alpha^{+} \mathrm{cDCs}$ (Figure 9b). These two molecules were indeed strongly induced in interfollicular cDCs of $9 \mathrm{~h} \mathrm{R} 848$-fed mice (Figure 9c,d). These data indicate that activated interfollicular cDCs have a contrasted pattern of gene expression that could lead to either stimulation or inhibition of T-cell activation.

\section{DISCUSSION}

In this study, we determined the genetic relationship and the location of $\mathrm{CDC}$ subsets in PP and analyzed their alteration upon a TLR7 agonist stimulation in vivo. We previously showed that $\mathrm{DN}$ and $\mathrm{CD} 11 \mathrm{~b}^{+}$dome cDCs are Batf3-independent and share some surface receptors such as SIRP $\alpha$ and Clec4a4. ${ }^{17}$ Here we extended these findings by showing that they belong to a unique SIRP $\alpha^{+}$cDC subset. Thus, our whole-genome expression analysis shows that they cluster together apart from $\mathrm{CD} 8 \alpha^{+}$cDCs, and that DN cDCs could correspond to a more immature differentiation state of SIRP $\alpha^{+}$cDCs than $\mathrm{CD} 11 \mathrm{~b}^{+}$ cDCs. In agreement with this transcriptional analysis, we identified putative transitional states between $\mathrm{DN}$ and $\mathrm{CD} 11 \mathrm{~b}^{+} \mathrm{cDCs}$ with progressive surface acquisition of MHCII, CD11b, EpCAM, and JAM-A.

Although it has been known for a long time that $\mathrm{CD} 11 \mathrm{c}^{+}$ $\mathrm{CD}_{11 b^{+}}$cells are located in the SED, ${ }^{14,35,36}$ the accurate identity of these phagocytes remained obscure, especially because three different dome phagocyte subsets with distinct origin express both integrins. ${ }^{17}$ The expression levels of CD11b differ between these phagocyte subsets ${ }^{17}$ and we show here that those that are actually detectable by microscopy in the SED with CD11b correspond to LysoDCs and some TIM- $4^{-}$LysoMacs but not $\mathrm{CD}_{11 \mathrm{~b}^{+}} \mathrm{cDCs}$. Accordingly, the main phagocyte populations of the SED are constituted of LysoDCs and TIM-4 ${ }^{-}$LysoMacs (Figure 10). In turn, CD $11 b^{+}$cDCs are mainly located in the IFR, although some of them may be present in the SED, especially at its base. Recently, Reboldi et $a l .{ }^{37}$ have reported that efficient IgA class switching of PP B cells requires their interaction with $\mathrm{CD} 11 \mathrm{c}^{\text {hi }} \mathrm{MHCII}{ }^{+} \mathrm{CD} 11 \mathrm{~b}^{+}$ cells in the SED. However, whether these $\mathrm{CD} 11 \mathrm{c}^{\mathrm{hi}} \mathrm{MHCII}^{+}$ $\mathrm{CD}_{11} \mathrm{~b}^{+}$phagocytes correspond to $\mathrm{CD} 11 \mathrm{~b}^{+} \mathrm{cDCs}$, LysoDCs, or LysoMacs remains to be established. Comparison of the ratio

Figure 9 Early synthesis of immunoregulatory molecules by PP cDCs in R848-fed mice. (a) Multiple gene set enrichment analysis of resting and activated PP phagocytes. BubbleGUM software was used as in Figure 1d to compare the expression of different gene signatures between resting (Rest) and R848-activated (Act) phagocyte subsets. Green boxes: resting LysoDCs and LysoMacs displayed a type I ISG signature as compared to resting cDCs. Blue box: type I ISGs were induced in activated as compared to resting cDCs. Orange box: migration and activation signatures were induced in cDCs upon R848 gavage. Black box: R848-activated cDCs displayed gene signatures involved both in activation $(+)$ and inhibition $(-)$ of the immune response. See Supplementary Table $\mathbf{S} 2$ for the list of gene signatures. (b) Normalized mean relative expression \pm s.d. of $C d 274$ and $P d c d 1 / g 2$ encoding the T-cell activation inhibitory ligands PD-L1 and PD-L2, respectively. (c,d) Confocal microscopy projection of IFR taken from C57BI/6 mice fed (bottom) or not (top) with R848 $9 \mathrm{~h}$ before collection and stained for CD11c (red), CD4 (cyan), CD8 (magenta), and either (c) PD-L1 or (d) PD-L2 (green). PD-L1 and PD-L2 expression is induced in CD11 ${ }^{+}$interfollicular cells of R848-fed mice. Bar, $20 \mu \mathrm{m}$. Act, activated; CDC, conventional dendritic cell; IFR, interfollicular region; ISG, interferon-stimulated gene; PP, Peyer's patch; Rest, resting. 

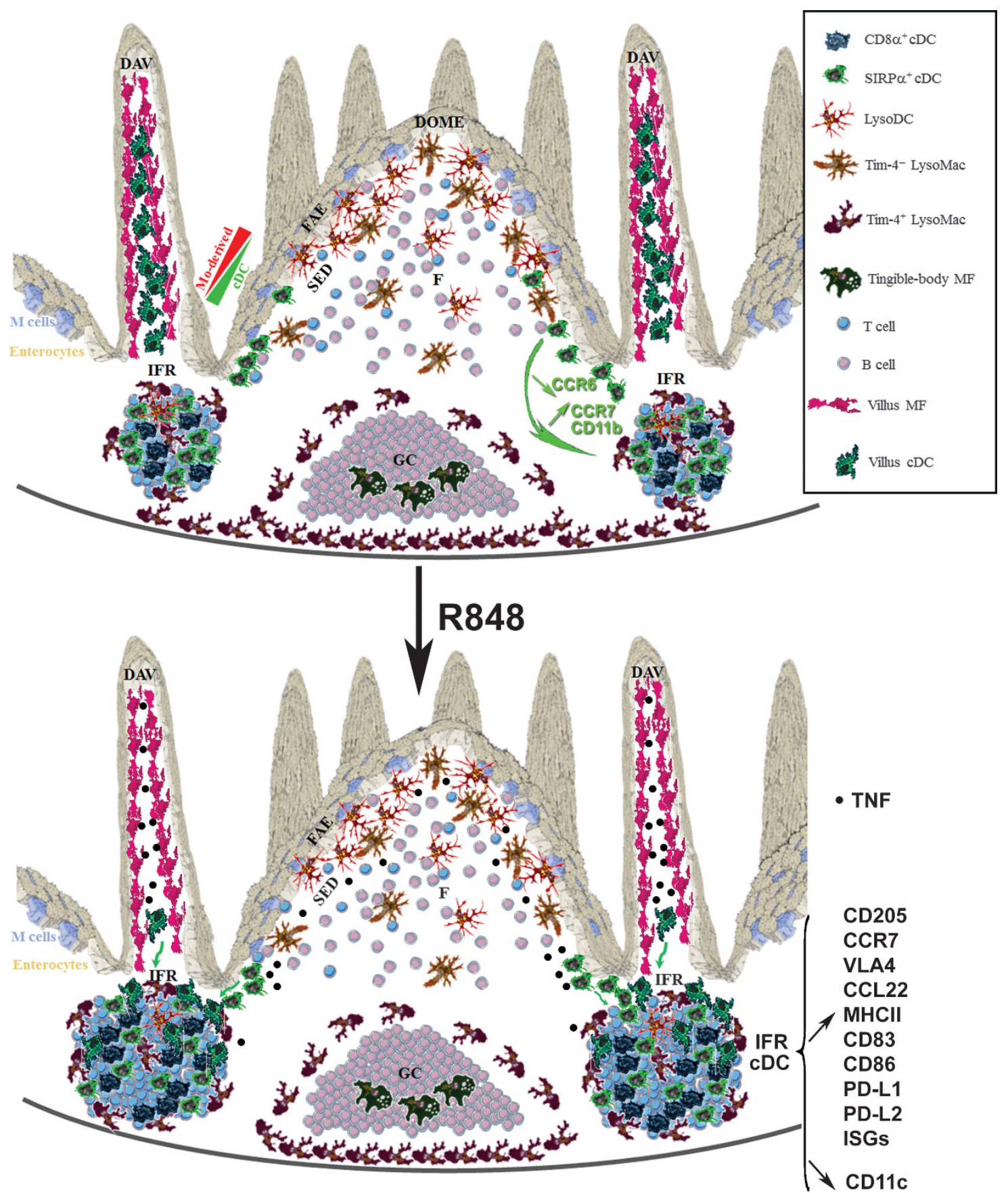

Figure 10 Model of mononuclear phagocyte system organization in PP at steady state and upon R848 stimulation. At steady state, the SED is mainly populated by monocyte-derived phagocytes, i.e., LysoDCs and TIM-4- LysoMacs, whereas the IFR is mainly populated by both SIRP $\alpha^{-}\left(\mathrm{CD} 8 \alpha^{+}\right)$and SIRP $\alpha^{+}$(mainly CD $11 b^{+}$) cDCs. However, monocyte-derived TIM-4 ${ }^{+}$macrophages are located in the IFR and few SIRP $\alpha^{+}$cDCs, likely DN cDCs, are located in the lower part of the SED and may migrate to the IFR by up- and downregulating CCR7 and CCR6, respectively, to become CD11b ${ }^{+}$cDCs. Upon R848 treatment, TLR7-activated cells, such as monocyte-derived cells and/or pDCs, produce TNF that in turn induces: (1) DAV cDC migration to the IFR; (2) CD8 $\alpha^{+}$cDC number increase; (3) CD205 expression in activated CD11b ${ }^{+}$CDCs of the IFR; (4) CCR7, VLA4, CCL22, MHCII, CD83, CD86, PD-L1, PD-L2, and ISGs upregulation and CD11c downregulation in all activated CDC. CDC, conventional dendritic cell; DAV, dome-associated villus; DN, double negative; F, follicle; FAE, follicle-associated epithelium; GC, germinal centre; PP, Peyer's patch; IFR, interfollicular region; ISGs, interferonstimulated genes; MF, macrophages; Mo-derived, monocyte-derived; SED, subepithelial dome.

of the different subsets obtained by flow cytometry with the ratio of the subsets that can be well defined with markers by microscopy suggests that DN cDCs are mainly located in the SED in agreement with their high and low expression of Ccr6 and $C c r 7$, respectively as compared to $\mathrm{CD} 11 \mathrm{~b}^{+}$dome cDCs.
We indeed found that CDCs of the IFR express CCR7, whereas those of the SED do not. Interestingly, CD $11 \mathrm{~b}^{+} \mathrm{CD} 11 \mathrm{c}^{+}$cells of the SED are known to be sensitive to CCL9 inhibition but not CCR6 deficiency, ${ }^{31}$ which is in agreement with the fact that LysoDCs and LysoMacs do not express Ccr6 but Ccr1. ${ }^{17}$ 
However, CCR6 may be required to recruit DN cDCs in the SED and the FAE, especially upon infection. ${ }^{38}$

R848 induces an indirect TNF-mediated activation of PP cDCs, which tends to attenuate each subset specificity while promoting common activation features, as previously observed on spleen cDCs after viral infection. ${ }^{5}$ Thus, key marker genes of each cDC subset are downregulated upon activation. Nevertheless, SIRP $\alpha$ expression stability during activation or during homeostatic maturation confirms that it is a much better marker of $\mathrm{CDC} 2$ than $\mathrm{CD} 11 \mathrm{~b}$. Upon activation, $\mathrm{CDCs}$ acquire the expression of innate defense genes that otherwise belong to the signature of PP monocyte-derived cells. This strongly supports the role of SED-located LysoDCs and TIM- $4^{-}$LysoMacs as the main constitutive first line of defense of PP. CD8 $8 \alpha^{+}$cDCs also rapidly acquire the expression of inhibitors of the T-cell response, such as PD-L1 and PD-L2, indicating that the immune response initiation is probably tightly regulated. This could also represent a way to favor naive T-cell priming rather than resident effector T-cell reactivation, as only some of the latter express the PD-L1 and PD-L2 receptor PD- $1 .^{39}$

Upon stimulation, SED DCs are believed to migrate from the SED to the IFR enriched in T cells in order to induce a mucosal adaptive immune response. This is supported by the fact that microsphere-loaded CD11c ${ }^{+}$cells normally located in the SED are observed in the IFR after cholera toxin or Salmonella Typhimurium feeding. ${ }^{8}$ In addition, systemic injection of soluble Toxoplasma gondii tachyzoite antigen induces a loss of CD11 $\mathrm{c}^{+}$ $\mathrm{CD}_{11 \mathrm{~b}^{+}}$cells in the SED and a concomitant recruitment of CD $11 \mathrm{c}^{+} \mathrm{CD} 11 \mathrm{~b}^{+}$cells in the IFR. ${ }^{14}$ Finally, the number of $\mathrm{CD} 103^{+}$cells supposed to be cDCs has been shown to increase in the IFR of R848-fed rats. ${ }^{12}$ Here we confirmed that cDC number rises in the IFR of mice upon R848 gavage and that all activated cDCs reside in the IFR as illustrated by their CD205, CD83, CD86, CD49d, CCL22, and CCR7 expression in this region. However, we also showed that this is at least in part due to $\mathrm{CD} 8 \alpha^{+}$interfollicular cDC number increase and to DAV cDC recruitment (Figure 10). The latter migration could allow in a single place a simultaneous comparison of antigens that have been sampled either in DAV or in SED. Taking into account that uptake of pathogens and toxins is largely favored in the FAE as compared to villus epithelium, ${ }^{18,19,40,41}$ such mechanism of antigen screening could help the mucosal immune system to distinguish innocuous from hazardous matters. It has now to be determined whether other stimuli than R848 induce similar migratory activities of DAV cDCs. If so, the current model of PP phagocyte activation will have to be refined and identity of migrating phagocytes to the IFR carefully assessed.

\section{METHODS}

Antibodies. Antibodies used are listed in Supplementary Information.

Animals. Six- to ten-week-old C57BL/6 mice were from Charles River Laboratories (Saint-Germain-Nuelles, France). Lys-EGFP and Cx3cr1GFP and Zbtb46-GFP mice have been previously described. ${ }^{42-44}$ All experiments were done in agreement with French and European guidelines for animal care.
Chemical treatments. Mice were injected intraperitoneal with EdU (Thermo Fisher Scientific Inc., Waltham, MA). For R848 in vivo experiments, mice were fed with $10 \mu \mathrm{g}$ of R848 (Invivogen, San Diego, CA, USA). An amount of $330 \mu \mathrm{g}$ rat anti-TNF-blocking antibody (clone MP6-XT22; BioLegend, San Diego, CA) or of isotype control (clone RTK2071) was given to mice intraperitoneal $30 \mathrm{~h}$ before R848.

PP cell extraction. PPs were digested for $40 \mathrm{~min}$ at room temperature with collagenase/DNase as previously described. ${ }^{45}$ All subsequent procedures were at $0-4{ }^{\circ} \mathrm{C}$. $\mathrm{CD} 11 \mathrm{c}^{+}$cells were sorted using antiCD11c microbeads and an AutoMACS magnetic cell separator according to the manufacturer's instructions (Miltenyi Biotec, Bergisch Gladbach, Germany).

Flow cytometry and cell sorting. $\mathrm{CD} 11 \mathrm{c}^{+}$cells were preincubated on ice for 10 min with the $2.4 \mathrm{G} 2$ antibody to block Fc receptors, stained for surface markers, and then permeabilized for lysozyme labeling according to the manufacturer's protocol (Intracellular staining kit; BD Biosciences, San Jose, CA). Cell viability was evaluated using Fixable Viability Dye eFluor 506 (eBiosciences, Thermo Fisher Scientific Inc.). Multiparameter flow cytometry and cell sorting were performed using a FACS LSRII and a FACSAria III (BD Biosciences), respectively. Data were analyzed with the BD FACSDiva software (BD Biosciences).

In vitro DC culture. Sorted PP DN and CD11b ${ }^{+}$DC subsets $\left(5 \times 10^{3}\right.$ cells) were immediately fixed (control) or cultured overnight in RPMI1640 supplemented with $10 \%$ fetal calf serum, $1 \%$ granulocytemacrophage colony-stimulating factor, $10 \%$ macrophage colonystimulating factor, $1 \%$ penicillin/streptomycin, $10 \mathrm{~mm}$ HEPES, $1 \mathrm{~mm}$ sodium pyruvate, $1 \mathrm{~mm}$ glutamine, $1 \mathrm{~mm}$ non-essential amino-acids, and $50 \mathrm{~mm}$ 2-ME. CD11b and MHCII surface expression were determined by flow cytometry as above.

RNA isolation and microarray analysis. The total RNA of PP-sorted phagocytes from three to five independent experiments was extracted with a RNAeasy PLUS micro kit (Qiagen, Hilden, Germany). Quantity, quality, and absence of genomic DNA contamination were assessed with a Bioanalyser (Agilent Technologies, Santa Clara, CA). Microarray experiments were performed by the Plateforme Biopuces of Strasbourg, France (http://www.igbmc.fr/technologies/5/team/54/) using the GeneChip Mouse Gene 1.0 ST array (Affymetrix, Thermo Fisher Scientific Inc.). Quality controls and normalization of array data were performed as previously described. ${ }^{45}$

Immunofluorescence staining and confocal microscopy. PPs of mice fed or not with R848 for 9 and 16 h were fixed with Antigenfix (Diapath, Martinengo, Italy) for $1 \mathrm{~h}$, washed and processed as previously described. ${ }^{18} \mathrm{PP}$ sections from anti-TNF-treated mice were first incubated with the Fab fragment of donkey anti-rat IgG (Jackson ImmunoResearch, West Grove, PA) to prevent the detection of rat anti-TNF with anti-rat secondary antibodies. Slides were observed with a Zeiss LSM 780 confocal microscope (Carl Zeiss, Oberkochen, Germany). Images were analyzed using Adobe Photoshop CS6 (Adobe systems, San Jose, CA).

Statistical analysis. Results were compared with GraphPad Prism 6 software (GraphPad Software, La Jolla, CA) using unpaired $t$-test with Welch's correction.

SUPPLEMENTARY MATERIAL is linked to the online version of the paper at http://www.nature.com/mi

\section{ACKNOWLEDGMENTS}

We thank the CIML histology, cytometry, and mouse house core facilities; Violaine Alunni and Christelle Thibault from the "Plateforme Biopuces et sequençage de I'IGBMC" (Strasbourg, France) for performing the microarray experiments; M. Barad for cell-sorting experiments; C. Jones, T. Soos, and C. Arendt for helpful discussions. We acknowledge the PICSL 
imaging facility of the CIML (Imaglmm), member of the national infrastructure France-Biolmaging supported by the French National Research Agency (ANR-10-INBS-04). This work was supported by institutional grants from INSERM, CNRS, and Aix-Marseille University to the CIML and by the I2HD collaborative project developed jointly by CIML and SANOFI. CDS was supported by the FRM fellowship FDT20160434982.

\section{AUTHOR CONTRIBUTION}

J.B. and H.L. designed the study. C.D.S., C.W., R.B. and J.B. performed all experiments. C.D.S., J.B. and H.L. analyzed and interpreted the data. H.L. wrote the manuscript. J.P.G., J.B. and C.D.S. contributed to the design of the study and revised the manuscript. L.C. performed cryostat sectioning. M.M., E.P. and M.D. analyzed the microarray data and gave feedback on the manuscript.

\section{DISCLOSURE}

The authors declare no conflict of interest.

\section{ACCESSION NUMBERS}

The microarray data have been deposited to the NCBI GEO under accession numbers GSE94380 and GSE65514.

(c) 2017 Society for Mucosal Immunology

\section{REFERENCES}

1. Banchereau, J. \& Steinman, R.M. Dendritic cells and the control of immunity. Nature 392, 245-252 (1998).

2. Reis e Sousa, C. Dendritic cells in a mature age. Nat. Rev. Immunol. 6, 476-483 (2006).

3. Trombetta, E.S. \& Mellman, I. Cell biology of antigen processing in vitro and in vivo. Annu. Rev. Immunol. 23, 975-1028 (2005).

4. Dalod, M., Chelbi, R., Malissen, B. \& Lawrence, T. Dendritic cell maturation: functional specialization through signaling specificity and transcriptional programming. EMBO J. 33, 1104-1116 (2014).

5. Manh, T.P., Alexandre, Y., Baranek, T., Crozat, K. \& Dalod, M. Plasmacytoid conventional, and monocyte-derived dendritic cells undergo a profound and convergent genetic reprogramming during their maturation. Eur. J. Immunol. 43, 1706-1715 (2013).

6. Cerovic, V., Bain, C.C., Mowat, A.M. \& Milling, S.W. Intestinal macrophages and dendritic cells: what's the difference?. Trends Immunol. 35, 270-277 (2014).

7. Anosova, N.G. et al. Cholera toxin, E. coli heat-labile toxin, and non-toxic derivatives induce dendritic cell migration into the follicle-associated epithelium of Peyer's patches. Mucosal Immunol. 1, 59-67 (2008).

8. Shreedhar, V.K., Kelsall, B.L. \& Neutra, M.R. Cholera toxin induces migration of dendritic cells from the subepithelial dome region to $T$ - and B-cell areas of Peyer's patches. Infect. Immun. 71, 504-509 (2003).

9. Chabot, S., Wagner, J.S., Farrant, S. \& Neutra, M.R. TLRs regulate the gatekeeping functions of the intestinal follicle-associated epithelium. J. Immunol. 176, 4275-4283 (2006).

10. Chabot, S.M. et al. TLR2 activation by proteosomes promotes uptake of particulate vaccines at mucosal surfaces. Vaccine 25, 5348-5358 (2007).

11. Chabot, S.M., Shawi, M., Eaves-Pyles, T. \& Neutra, M.R. Effects of flagellin on the functions of follicle-associated epithelium. J. Infect. Dis. 198, 907-910 (2008).

12. Yrlid, U., Milling, S.W., Miller, J.L., Cartland, S., Jenkins, C.D. \& MacPherson, G.G. Regulation of intestinal dendritic cell migration and activation by plasmacytoid dendritic cells, TNF-alpha and type 1 IFNs after feeding a TLR7/8 ligand. J. Immunol. 176, 5205-5212 (2006).

13. Vremec, D. \& Shortman, K. Dendritic cell subtypes in mouse lymphoid organs: cross-correlation of surface markers, changes with incubation, and differences among thymus, spleen, and lymph nodes. J. Immunol. 159, 565-573 (1997).

14. Iwasaki, A. \& Kelsall, B.L. Localization of distinct Peyer's patch dendritic cell subsets and their recruitment by chemokines macrophage inflammatory protein (MIP)-3alpha, MIP-3beta, and secondary lymphoid organ chemokine. J. Exp. Med. 191, 1381-1394 (2000).
15. Iwasaki, A. \& Kelsall, B.L. Unique functions of CD11b,+ CD8 alpha + , and double-negative Peyer's patch dendritic cells. J. Immunol. 166, 4884-4890 (2001).

16. Anjuere, F. et al. Definition of dendritic cell subpopulations present in the spleen, Peyer's patches, lymph nodes, and skin of the mouse. Blood 93, 590-598 (1999).

17. Bonnardel, J. et al. Innate and adaptive immune functions of Peyer's patch monocyte-derived cells. Cell Rep. 11, 770-784 (2015).

18. Lelouard, H., Fallet, M., de Bovis, B., Meresse, S. \& Gorvel, J.P. Peyer's patch dendritic cells sample antigens by extending dendrites through $\mathrm{M}$ cell-specific transcellular pores. Gastroenterology 142, 592-601, e593 (2012).

19. Lelouard, H. et al. Pathogenic bacteria and dead cells are internalized by a unique subset of Peyer's patch dendritic cells that express lysozyme. Gastroenterology 138, 173-184 e171-173 (2010).

20. Yamakita, Y. et al. Fascin1 promotes cell migration of mature dendritic cells. J. Immunol. 186, 2850-2859 (2011).

21. Fukao, T., Frucht, D.M., Yap, G., Gadina, M., O'Shea, J.J. \& Koyasu, S. Inducible expression of Stat4 in dendritic cells and macrophages and its critical role in innate and adaptive immune responses. J. Immunol. 166, 4446-4455 (2001).

22. Spinelli, L., Carpentier, S., Montanana Sanchis, F., Dalod, M. \& Vu Manh, T.P. BubbleGUM: automatic extraction of phenotype molecular signatures and comprehensive visualization of multiple gene set enrichment analyses. BMC Genomics 16, 814 (2015).

23. Baranek, T. et al. Differential responses of immune cells to type I interferon contribute to host resistance to viral infection. Cell Host Microbe 12, 571-584 (2012).

24. Gautier, E.L. et al. Gene-expression profiles and transcriptional regulatory pathways that underlie the identity and diversity of mouse tissue macrophages. Nat. Immunol. 13, 1118-1128 (2012).

25. Grajales-Reyes, G.E. et al. Batf3 maintains autoactivation of Iff8 for commitment of a CD8alpha $(+)$ conventional DC clonogenic progenitor. Nat. Immunol. 16, 708-717 (2015).

26. Miller, J.C. etal. Deciphering the transcriptional network of the dendritic cell lineage. Nat. Immunol. 13, 888-899 (2012).

27. Tamoutounour, S. et al. Origins and functional specialization of macrophages and of conventional and monocyte-derived dendritic cells in mouse skin. Immunity 39, 925-938 (2013).

28. Vu Manh, T.P. et al. Defining mononuclear phagocyte subset homology across several distant warm-blooded vertebrates through comparative transcriptomics. Front. Immunol. 6, 299 (2015).

29. Xue, J. et al. Transcriptome-based network analysis reveals a spectrum model of human macrophage activation. Immunity 40, 274-288 (2014).

30. Dieu, M.C. et al. Selective recruitment of immature and mature dendritic cells by distinct chemokines expressed in different anatomic sites. J. Exp. Med. 188, 373-386 (1998).

31. Zhao, X. et al. CCL9 is secreted by the follicle-associated epithelium and recruits dome region Peyer's patch CD11b + dendritic cells. J. Immunol. 171, 2797-2803 (2003).

32. Gunn, M.D., Tangemann, K., Tam, C., Cyster, J.G., Rosen, S.D. \& Williams, L.T. A chemokine expressed in lymphoid high endothelial venules promotes the adhesion and chemotaxis of naive T lymphocytes. Proc. Natl Acad. Sci. USA 95, 258-263 (1998).

33. Ngo, V.N., Tang, H.L. \& Cyster, J.G. Epstein-Barr virus-induced molecule 1 ligand chemokine is expressed by dendritic cells in lymphoid tissues and strongly attracts naive T cells and activated B cells. J. Exp. Med. 188, 181-191 (1998).

34. Willimann, K. et al. The chemokine SLC is expressed in Tcell areas of lymph nodes and mucosal lymphoid tissues and attracts activated T cells via CCR7. Eur. J. Immunol. 28, 2025-2034 (1998).

35. Cook, D.N. et al. CCR6 mediates dendritic cell localization, lymphocyte homeostasis, and immune responses in mucosal tissue. Immunity $\mathbf{1 2}$ 495-503 (2000).

36. Varona, R. et al. CCR6-deficient mice have impaired leukocyte homeostasis and altered contact hypersensitivity and delayed-type hypersensitivity responses. J. Clin. Invest. 107, R37-R45 (2001).

37. Reboldi, A., Arnon, T.I., Rodda, L.B., Atakilit, A., Sheppard, D. \& Cyster, J.G. IgA production requires $B$ cell interaction with subepithelial dendritic cells in Peyer's patches. Science 352, aaf4822 (2016). 


\section{ARTICLES}

38. Salazar-Gonzalez, R.M. et al. CCR6-mediated dendritic cell activation of pathogen-specific T cells in Peyer's patches. Immunity 24, 623-632 (2006).

39. Ugur, M., Schulz, O., Menon, M.B., Krueger, A. \& Pabst, O. Resident CD4 + T cells accumulate in lymphoid organs after prolonged antigen exposure. Nat. Commun. 5, 4821 (2014).

40. Rios, D., Wood, M.B., Li, J., Chassaing, B., Gewirtz, A.T. \& Williams, I.R. Antigen sampling by intestinal $M$ cells is the principal pathway initiating mucosal IgA production to commensal enteric bacteria. Mucosal Immunol. 9, 907-916 (2016).

41. Schulz, O. \& Pabst, O. Antigen sampling in the small intestine. Trends Immunol. 34, 155-161 (2013).
42. Jung, S. et al. Analysis of fractalkine receptor CX(3)CR1 function by targeted deletion and green fluorescent protein reporter gene insertion. Mol. Cell Biol. 20, 4106-4114 (2000).

43. Faust, N., Varas, F., Kelly, L.M., Heck, S. \& Graf, T. Insertion of enhanced green fluorescent protein into the lysozyme gene creates mice with green fluorescent granulocytes and macrophages. Blood 96, 719-726 (2000).

44. Satpathy, A.T. et al. Zbtb46 expression distinguishes classical dendritic cells and their committed progenitors from other immune lineages. J. Exp. Med. 209, 1135-1152 (2012).

45. Bonnardel, J., Da Silva, C., Masse, M., Montañana-Sanchis, F., Gorvel, J.-P. \& Lelouard, H. Gene expression profiling of the Peyer's patch mononuclear phagocyte system. Genomics Data 5, 21-24 (2015). 